\title{
On the Frequency Domain Accuracy of Closed Loop Estimates
}

\author{
Brett Ninness* $\quad$ Håkan Hjalmarsson $^{\dagger}$
}

\begin{abstract}
It has been argued that the frequency domain accuracy of high model-order estimates obtained on the basis of closed loop data is largely invariant to whether direct or indirect approaches are used. The analysis underlying this conclusion has employed variance expressions that are asymptotic both in the data length and the model order, and hence are approximations when either of these are finite. However, recent work has provided variance expressions that are exact for finite (possibly low) model order, and hence can potentially deliver more accurate quantification of estimation accuracy. This paper revisits the study of identification from closed loop data in light of these new quantifications and establishes that, under certain assumptions of white spectra, there can be significant differences in the accuracy of frequency response estimates that are dependent on what type of direct, indirect or joint input-output identification strategy is pursued.
\end{abstract}

\section{Introduction}

Over the last several years there has been significant interest in the interplay between system identification and subsequent control design [2, 3, 5, 13]. In particular, a variety of estimation techniques adapted to these closed and open loop scenarios have been developed. They can be broadly divided into 'direct', 'indirect' and 'joint input-output' estimation methods, and of significant relevance to this paper has been recent work $[1,8,4,13]$ examining the relative accuracy of estimates obtained via the different approaches.

In particular [1,4] uses certain approximations derived in [9] for the mean square error in frequency response estimates, and whose accuracy depends on data length and model order being large. Via these $[1,4]$ then argues that, in the limit as the model order tends to infinity, direct, indirect and joint input-output methods offer the same accuracy in the frequency domain. However, in [4] the authors point out that an assumption of large model order 'apparently diminishes possible differences'. Furthermore, in [13] it is noted that 'this asymptotic variance analysis tool is also quite crude', and 'for finite model orders, the variance results will likely become different over the several methods'. Indeed this expected difference is established in [1] for parameter estimates, but not for the associated frequency response estimates.

This prior work and observations by other authors suggest the need for further study that quantifies the frequency domain accuracy of direct, indirect and joint input-output estimates that are valid for finite, and possibly low model order. Such is the purpose of this paper, which addresses the problem

${ }^{*}$ This work was supported by the Australian Research Council. This author is with the School of of Electrical Engineering \& Computer Science, University of Newcastle, Australia and can be contacted at email:brett@ee. newcastle.edu. au or FAX: +61 49216993.

${ }^{\dagger}$ This author is with the Department of Sensors, Signals and Systems (Automatic Control), The Royal Institute of Technology, S-100 44 Stockholm, Sweden and may be contacted at email:hakan.hjalmarsson@s3.e.kth. se or FAX: +4687907329 
by using new expressions for the mean square estimation error that have been recently developed in [10], and whose accuracy is not dependent on the model order being large; they are exact for finite model order.

As will be seen, these exact expressions can, depending on the estimation conditions, be very different to those arising from [9] and employed in [1, 8, 4], and this will imply new conclusions on the relationship between direct, indirect and joint input-output estimation approaches.

For example, under certain assumptions on reference spectrum and control design, we establish that for finite model order, the variance of the estimated frequency response arising from a direct method can be very different to that associated with indirect and joint input-output methods.

Furthermore, we establish that, again in the frequency domain and for finite model order, the variance of these flavours of indirect and joint-input output estimates are none of them equivalent to one another. An exception to this conclusion is found in the special case of simple proportional control, where this paper establishes that the variances of direct and some indirect and joint inputoutput approaches are equal, provided that the reference input to measurement noise SNR is large.

An overview of the organisation of this paper, is that in the following $\S 2$ the assumed closed loop data collection scenario is detailed, with $\S 3$ then describing the direct prediction-error estimation method studied in this paper together with the key technical result of Theorem 3.1 that allows for accurate variance error quantification, and is the basis for all subsequent results. The subsequent $\S 4$ then contains the main results of the paper, with $\S 4.2 .1-\S 4.2 .3$ examining the various indirect methods, and $\S 4.3 .1-\S 4.3 .2$ studing joint input-output methods. Simulation examples illustrating the results and conclusions in these sections are provided in $\S 5$. A final $\S 6$ provides some concluding remarks, with subsequent appendices containing technical material too lengthy to be contained in the main body of the paper.

\section{Preliminaries}

This paper addresses the closed loop control scenario depicted in Figure 1 of

$$
\begin{aligned}
\mathcal{S}: \quad y_{t} & =G(q) u_{t}+\nu_{t}, \quad \nu_{t}=H(q) e_{t} \\
u_{t} & =K(q)\left[r_{t}-y_{t}\right]
\end{aligned}
$$

for some underlying true system $\mathcal{S}$ characterised by the transfer functions $G(q)$ and $H(q)$ and which is under the influence of a linear time invariant controller $K(q)$ and an external set point signal $\left\{r_{t}\right\}$. Here $G(q), H(q)$ and $K(q)$ are all rational in the backward shift operator $q^{-1}$ and $\left\{e_{t}\right\}$ is a zero-mean white noise sequence that satisfies $\mathbf{E}\left\{e_{t}^{2}\right\}=\sigma^{2}, \mathbf{E}\left\{\left|e_{t}\right|^{8}\right\}<\infty$. In this case, the relationship between

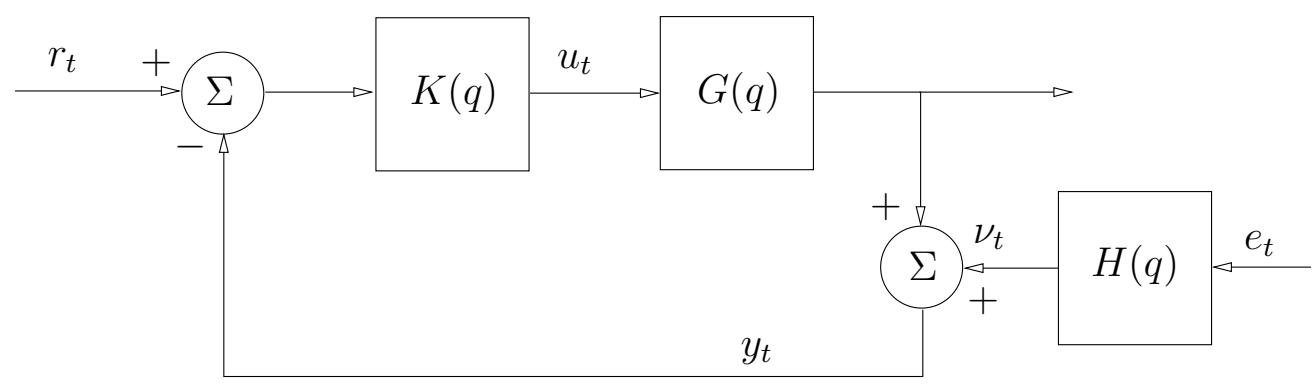

Figure 1: Closed Loop Control Scenario considered in this paper 
the signals in (1), (2) may also be expressed as

$$
\begin{aligned}
& y_{t}=T(q) r_{t}+S(q) e_{t} \\
& u_{t}=S(q) K(q) r_{t}-S(q) K(q) e_{t}
\end{aligned}
$$

where $S(q)$ and $T(q)$ are the sensitivity and complementary sensitivity functions given (respectively) as

$$
S(q)=\frac{1}{1+G(q) K(q)}, \quad T(q)=\frac{G(q) K(q)}{1+G(q) K(q)} .
$$

It will be assumed that the reference signal $\left\{r_{t}\right\}$ is a quasi-stationary process with associated spectral density $\Phi_{r}(\omega)$, in which case provided the poles of $S(q) K(q)$ are in the open unit disk, then other spectral densities will exist such as

$$
\Phi_{u}(\omega)=\left|K\left(e^{j \omega}\right) S\left(e^{j \omega}\right)\right|^{2} \Phi_{r}(\omega)+|K S H|^{2} \sigma^{2}, \quad \Phi_{u e}(\omega)=-S K H \sigma^{2} .
$$

Of particular interest will be the spectral density $\Phi_{u}^{r}(\omega)$ of the component of $\left\{u_{t}\right\}$ that derives solely from $\left\{r_{t}\right\}$. Via (4) this is given as

$$
\Phi_{u}^{r}(\omega)=\left|K\left(e^{j \omega}\right) S\left(e^{j \omega}\right)\right|^{2} \Phi_{r}(\omega) .
$$

However, it will be useful to recognise that via substitution of the expression arising from (4) for $\Phi_{u e}(\omega)$, the component not due to $\left\{r_{t}\right\}$ may be subtracted from the expression for $\Phi_{u}(\omega)$ to yield the alternate formulation

$$
\Phi_{u}^{r}(\omega)=\Phi_{u}(\omega)-\frac{\left|\Phi_{u e}(\omega)\right|^{2}}{\sigma^{2}} .
$$

A prime focus of this paper will be the provision of quantifications that are exact with respect to a finite model order of interest. For this purpose, it will be necessary to restrict the class of possible controllers, such that if $G(q)$ and $K(q)$ are of rational form

$$
G(q)=\frac{B(q)}{A(q)}, \quad K(q)=K \frac{P(q)}{L(q)}
$$

where $K \in \mathbf{R}$ and $A(q), B(q), L(q), P(q)$ are all polynomials in $q^{-1}$, then it will be required that the numerator $P(q)$ is formed as a subset of the open loop poles $A(q)$. That is, for some polynomial $\widetilde{A}(q)$ in $q^{-1}$

$$
\widetilde{A}(q) P(q)=A(q) \text {. }
$$

It is worth noting that an important subclass of this set of controllers are those of the pole-cancelling sort, in which $P(q)=A(q), \widetilde{A}(q)=1$ is chosen.

This, and further specialised requirements that are necessary to impose in the interests of dealing with finite model order are collected in the following set of standing assumptions.

Standing Assumptions 2.1. The following standing assumptions will be repeatedly imposed:

1. $\Phi_{r}(\omega)=\mu$ a constant;

2. The controller $K(q)$ is of the form

$$
K(q)=K \frac{P(q)}{L(q)} \quad L(q)=\prod_{k=1}^{m_{\ell}}\left(1-\ell_{k} q^{-1}\right), \quad K \in \mathbf{R} ;
$$

and where $P(q)$ satisfies (9); 
3. The closed loop poles are the zeros of

$$
A_{c}(z)=\widetilde{A}(z) L(z)+K B(z)
$$

4. The true noise model $H(q)=1$, and the model structure is chosen such that $\varepsilon_{t}\left(\theta_{\circ}\right)=e_{t}$ (ie. there is no undermodelling) and such that there are no pole-zero cancellations in any of the limiting transfer functions parameterised by $\theta_{\circ}$ (ie. the system state dimension is not overmodelled);

5. The underlying true system $G(q)$ is asymptotically stable.

Assumption point 4 is a formal statement that, whatever model structure is used, it is of sufficient order to encompass whatever underlying true system is relevant, while at the same time not involving an over-parameterisation beyond the McMillan degree of any dynamics or noise model concerned.

In what follows, the implied necessary model order of input-output dynamics will be explicitly stated, since it will have a very direct bearing on variance quantifications. However, since the same is not true for the noise model, then in the interests of conciseness it will simply be assumed from now on that it is sufficiently flexible that point 4 is satisfied.

\section{Direct Identification}

This paper addresses the issue of estimating the dynamics $G(q)$ on the basis of observing $N$ observations of input-output response. For this purpose, the paper considers the use of a model structure

$$
\mathcal{M}: \quad y_{t}=G(q, \theta) u_{t}+H(q, \theta) e_{t}=\frac{B(q, \theta)}{A(q, \theta)} u_{t}+\frac{C(q, \theta)}{D(q, \theta)} e_{t}
$$

where

$$
\begin{aligned}
& A(q, \theta)=1+a_{1} q^{-1}+a_{2} q^{-1}+\cdots+a_{m_{a}} q^{-m_{a}}, \\
& B(q, \theta)=b_{1} q^{-1}+b_{2} q^{-1}+\cdots+b_{m_{b}} q^{-m_{b}}, \\
& D(q, \theta)=1+d_{1} q^{-1}+d_{2} q^{-1}+\cdots+d_{m_{d}} q^{-m_{d}}, \\
& C(q, \theta)=1+c_{1} q^{-1}+c_{2} q^{-1}+\cdots+c_{m_{c}} q^{-m_{c}},
\end{aligned}
$$

for some integers $m_{a}, m_{b}, m_{c}, m_{d}$ and $\theta \in \mathbf{R}$ is a vector parameterising the above polynomials according to

$$
\theta=\left[a_{1}, \cdots, a_{m_{a}}, b_{1}, \cdots, b_{m_{b}}, c_{1}, \cdots, c_{m_{c}}, d_{1}, \cdots, d_{m_{d}}\right]^{T} .
$$

Under a scheme of so-called 'direct' identification, one works directly with the signals $\left\{y_{t}\right\}$ and $\left\{u_{t}\right\}$ so that the dynamics in (11) are estimated by forming $\widehat{\theta}_{N}$ according to

$$
\widehat{\theta}_{N}=\underset{\theta \in \mathbf{R}}{\arg \min } V_{N}(\theta), \quad V_{N}(\theta) \triangleq \frac{1}{2 N} \sum_{t=1}^{N} \varepsilon_{t}^{2}(\theta)
$$

where

$$
\varepsilon_{t}(\theta)=y_{t}-\widehat{y}_{t}(\theta)
$$


is the error between the observed $y_{t}$ and the mean square optimal one step ahead prediction of $y_{t}$, according to the model (11) given as

$$
\widehat{y}_{t}(\theta)=H^{-1}(q, \theta) G(q, \theta) u_{t}+\left[1-H^{-1}(q, \theta)\right] y_{t} .
$$

The accuracy of this estimation strategy can be assessed by noting that, under the assumption that the true system $\mathcal{S}$ given in (1) is contained within the model set $\mathcal{M}$ given by (11) for some parameter value $\theta_{\circ}^{n}$, then with probability one $[9,7]$

$$
\lim _{N \rightarrow \infty} \widehat{\theta}_{N}=\theta_{\circ}, \quad \varepsilon_{t}\left(\theta_{\circ}\right)=e_{t}
$$

and furthermore, in the case where the model orders satisfy

$$
m=m_{a}=m_{b}=m_{c}=m_{d}
$$

then, as established in [9], under certain assumptions on the use of a regularised estimate, and with $\Phi_{\nu}(\omega)$ denoting the power spectral density of $\left\{\nu_{t}\right\}$

$$
\begin{aligned}
\lim _{m \rightarrow \infty} \lim _{N \rightarrow \infty} \operatorname{Cov}\left\{\left[\begin{array}{c}
G\left(e^{j \omega}, \widehat{\theta}_{N}\right) \\
H\left(e^{j \omega}, \widehat{\theta}_{N}\right)
\end{array}\right]\right\} & =\Phi_{\nu}(\omega)\left[\begin{array}{cc}
\frac{\Phi_{u}(\omega)}{\Phi_{u e}(\omega)} & \Phi_{u e}(\omega) \\
\sigma^{2}
\end{array}\right]^{-1} \\
& =\frac{\Phi_{\nu}(\omega)}{\sigma^{2} \Phi_{u}(\omega)-\left|\Phi_{u e}(\omega)\right|^{2}}\left[\begin{array}{cc}
\frac{\sigma^{2}}{\Phi_{u e}(\omega)} & -\Phi_{u e}(\omega) \\
-\Phi_{u}(\omega)
\end{array}\right] .
\end{aligned}
$$

Commonly, this result has been employed by assuming that the convergence in (17) has approximately occurred, in which case

$$
\operatorname{Var}\left\{G\left(e^{j \omega}, \widehat{\theta}_{N}\right)\right\} \approx \frac{m}{N} \frac{\Phi_{\nu}(\omega)}{\Phi_{u}(\omega)-\left|\Phi_{u e}(\omega)\right|^{2} / \sigma^{2}}=\frac{m}{N} \frac{\Phi_{\nu}(\omega)}{\Phi_{u}^{r}(\omega)}
$$

is taken as a useful quantification of estimation accuracy.

However, in the interests of improving this accuracy, the recent work [10] has established results that are exact for finite model order $m$. In particular, the following result is central to the developments of this paper.

Theorem 3.1. Consider the case of estimation using the model structure (11)-(14) using the prediction error approach (16), (3). Suppose that $\Phi_{u}(\omega)$ has spectral factorisation

$$
\Phi_{u}(\omega)=\mu\left|F\left(e^{j \omega}\right)\right|^{2}
$$

where $F(z)$ and $F^{-1}(z)$ are both monic and analytic outside the unit disk, and suppose that $A_{\dagger}(z)$ given by

$$
A_{\dagger}(z)=A^{2}\left(z, \theta_{\circ}\right) \frac{H\left(z, \theta_{\circ}\right)}{F(z)}
$$

is a polynomial in $z^{-1}$ of order no greater than $m_{a}+m_{b}$. In this case, define $\left\{\xi_{1}, \cdots, \xi_{m_{a}+m_{b}}\right\}$ to be the zeros of $z^{m_{a}+m_{b}} A_{\dagger}(z)$ and $\left\{\eta_{1}, \cdots, \eta_{m_{c}+m_{d}}\right\}$ to be the zeros of $D\left(z, \theta_{\circ}\right) C\left(z, \theta_{\circ}\right)$ and use them to further define the functions

$$
\kappa(\omega) \triangleq \sum_{k=1}^{m_{a}+m_{b}} \frac{1-\left|\xi_{k}\right|^{2}}{\left|e^{j \omega}-\xi_{k}\right|^{2}}, \quad \widetilde{\kappa}(\omega) \triangleq \sum_{k=1}^{m_{c}+m_{d}} \frac{1-\left|\eta_{k}\right|^{2}}{\left|e^{j \omega}-\eta_{k}\right|^{2}} .
$$


Then, provided that neither of $G\left(z, \theta_{\circ}\right)$ or $H\left(z, \theta_{\circ}\right)$ contain any pole-zero cancellations, and that $\Phi_{u e}(\omega)=0$

$$
\lim _{N \rightarrow \infty} N \operatorname{Cov}\left\{\left[\begin{array}{c}
G\left(e^{j \omega}, \widehat{\theta}_{N}\right) \\
H\left(e^{j \omega}, \widehat{\theta}_{N}\right)
\end{array}\right]\right\}=\left|H\left(e^{j \omega}, \theta_{\circ}\right)\right|^{2}\left[\begin{array}{cc}
\frac{\sigma^{2}}{\Phi_{u}(\omega)} \kappa(\omega) & 0 \\
0 & \widetilde{\kappa}(\omega)
\end{array}\right] .
$$

Furthermore, if the model structure is restricted to be an Output-Error one in which $H\left(q, \theta^{n}\right)=1$ is fixed, then the assumption of $\Phi_{u e}(\omega)=0$ can be dropped, and

$$
\lim _{N \rightarrow \infty} N \operatorname{Cov}\left\{G\left(e^{j \omega}, \widehat{\theta}_{N}\right)\right\}=\frac{\sigma^{2}}{\Phi_{u}(\omega)} \kappa(\omega) .
$$

Proof. See [10].

This result suggests the quantification

$$
\mathbf{E}\left\{\left|G\left(e^{j \omega}, \widehat{\theta}_{N}\right)-G\left(e^{j \omega}, \theta_{\circ}\right)\right|^{2}\right\} \approx \frac{1}{N} \frac{\Phi_{\nu}(\omega)}{\Phi_{u}(\omega)} \sum_{k=1}^{m_{a}+m_{b}} \frac{1-\left|\xi_{k}\right|^{2}}{\left|e^{j \omega}-\xi_{k}\right|^{2}}
$$

Since it is derived from (21) (which unlike (17) does not depend on a model order $m$ tending to infinity) then unlike (18), the accuracy of the quantification (22) does not depend on the model order being large and hence (as will be illustrated here), it is likely to be more informative for realistic finite and/or low model orders. However, it is also important to emphasise that although the accuracy of (22) does not depend on high model order, it is still valid for arbitrarily high model order subject to the restriction of no pole zero cancellations in $G\left(z, \theta_{\circ}\right)$ or $H\left(z, \theta_{\circ}\right)$.

Moreover, by virtue of the frequency dependent term $\kappa(\omega)$, the quantification (22) can be quantitatively and qualitatively very different to (18). Therefore, any previous conclusions made on the basis of (18) may need to be significantly modified in cases where (22) provides a more reliable and at the same time rather different quantification. In particular, it is appropriate to revisit any debate on the equivalence (or not) of direct versus indirect versus joint input-output estimates that was made on the basis of (18).

\section{Main Results}

As pointed out in [1], provided a model structure (11) is employed such that $G(q, \theta), H(q, \theta)$ are both of sufficient order to describe the underlying true system $\mathcal{S}$, then the direct approach coupled with the prediction error algorithm [7] will provide a consistent estimate. Moreover, as argued in [1, 4], in this same situation the quantification (18) yields quantification of estimation accuracy, valid in the asymptotic limit as model order tends to infinity, and which can also be used as a basis for computing the mean square error of certain indirect and joint input-output estimation methods, principally via a first order Taylor approximation argument.

In the following sections, this latter issue, and high model order analysis of it presented in $[1,4,13]$ is revisited in an alternative context of finite, and perhaps low model order, and in the light of the new result of Theorem 3.1 . 


\subsection{Direct Identification}

The main result for the case of direct estimation as described in $\S 3$ can now be presented without further preliminaries, save to mention that in what follows, a direct estimate formed from the model structure (11) will be denoted as $G_{\mathrm{di}}\left(q, \widehat{\theta}_{N}\right)$.

Theorem 4.1. Suppose that the prediction error estimation method outlined previously is employed and that the standing assumptions 2.1 are satisfied. Suppose further that the polynomial $A(z) A_{c}(z)$ is of order less than or equal to the sum of the numerator and denominator model orders $m_{a}+m_{b}$. Then

$$
\lim _{N \rightarrow \infty} N \operatorname{Var}\left\{G_{\mathrm{di}}\left(e^{j \omega}, \widehat{\theta}_{N}\right)\right\}=\frac{\sigma^{2}}{\Phi_{u}(\omega)} \cdot \kappa_{\mathrm{di}}(\omega)
$$

where $\kappa_{\mathrm{di}}(\omega)$ is given by (20) with the associated zeros $\left\{\xi_{1}, \cdots, \xi_{m_{a}+m_{b}}\right\}$ being defined as those of the polynomial $z^{m_{a}+m_{b}} A(z) A_{c}(z)$.

Proof. See Appendix A.1

This result suggests the following quantification whose accuracy depends on data length $N$ being large, but which is exact for the indicated finite model order

$$
\operatorname{Var}\left\{G_{\mathrm{di}}\left(e^{j \omega}, \widehat{\theta}_{N}\right)\right\} \approx \frac{\sigma^{2}}{N \Phi_{u}(\omega)} \kappa_{\mathrm{di}}(\omega)
$$

This expression will be closely examined in the following sections as a means of comparing and contrasting the frequency domain accuracy of direct estimates as quantified by (24), and various indirect and joint input-output methods.

However, before proceeding to this discussion, it is worth noting that a central point underlying this and subsequent results is that the estimation problem can be decomposed according to various choices of dynamics and pre-filter. For example, with regard to a pre-filter $X(q)$, the model structure (11) can be re-written as

$$
y_{t}=G_{x}(q, \theta) x_{t}+H(q, \theta) e_{t}, \quad G_{x}(q, \theta) \triangleq G(q, \theta) X^{-1}(q), \quad x_{t} \triangleq X(q) u_{t} .
$$

Therefore, one could consider a generalised version of the direct identification scenario in which a model structure $G_{x}(q, \theta)$ was estimated on the basis of the residual

$$
\varepsilon_{t}(\theta)=H^{-1}(q, \theta)\left[y_{t}-G_{x}(q, \theta) u_{x}(t)\right]
$$

and then an estimate $G_{\operatorname{dix}}\left(q, \widehat{\theta}_{N}\right)$ of the dynamics $G(q)$ was obtained as

$$
G_{\operatorname{di} x}\left(q, \widehat{\theta}_{N}\right)=G_{x}\left(q, \widehat{\theta}_{N}\right) X(q) .
$$

The question then arises as to the accuracy of $G_{\mathrm{di} x}\left(q, \widehat{\theta}_{N}\right)$ relative to $G_{\mathrm{di}}\left(q, \widehat{\theta}_{N}\right)$. The asymptotic in model order theory leading to (18) suggests that the two ingredients of

$\operatorname{Var}\left\{G_{x}\left(e^{j \omega}, \widehat{\theta}_{N}\right)\right\} \approx \frac{m}{N} \frac{\Phi_{\nu}(\omega)}{\left|X\left(e^{j \omega}\right)\right|^{2} \Phi_{u}(\omega)}, \quad \operatorname{Var}\left\{G_{\text {dix }}\left(e^{j \omega}, \widehat{\theta}_{N}\right)\right\}=\left|X\left(e^{j \omega}\right)\right|^{2} \operatorname{Var}\left\{G_{x}\left(e^{j \omega}, \widehat{\theta}_{N}\right)\right\}$

can be combined to conclude that

$$
\operatorname{Var}\left\{G_{\mathrm{di} x}\left(e^{j \omega}, \widehat{\theta}_{N}\right)\right\} \approx \operatorname{Var}\left\{G_{\mathrm{di}}\left(e^{j \omega}, \widehat{\theta}_{N}\right)\right\} \approx \frac{m}{N} \frac{\Phi_{\nu}(\omega)}{\Phi_{u}(\omega)}
$$


and hence suggest that pre-filtering does not affect estimation accuracy. Central to the results of this paper is that, while this conclusion may be valid in the asymptotic model order case, it is not generally true for the practically important finite model order case. See also the recent work [14] for a discussion of this point.

In particular, it is straightforward to establish via the methods of the proof of Theorem 4.2, that under the same assumptions of the previous Theorem 4.2 pertaining to direct identification, but with the model orders of $G_{x}\left(q, \theta^{n}\right)$ increased to accommodate the presence of the $X^{-1}(q, \theta)$ component

$$
\lim _{N \rightarrow \infty} N \operatorname{Var}\left\{G_{\operatorname{dix}}\left(e^{j \omega}, \widehat{\theta}_{N}\right)\right\}=\frac{\sigma^{2}}{\Phi_{u}(\omega)} \kappa_{\operatorname{di} x}(\omega)
$$

where $\kappa_{\mathrm{di} x}(\omega)$ is given by (20) with the $\left\{\xi_{k}\right\}$ in that expression being the zeros of

$$
z^{m_{a}+m_{b}} A(z) A_{c}(z) X_{N}(z) X_{D}(z), \quad \text { where } \quad X(q)=\frac{X_{N}(q)}{X_{D}(q)} .
$$

Clearly, comparing (28) with (23) indicates that in the finite model order case, pre-filtering can have a substantial effect on estimation accuracy, according to what might be chosen for the poles and zeros of the pre-filter $X(q)$. This, combined with the fact that indirect estimation methods can be viewed as the introduction of particular pre-filters [1], is central to the results here.

\subsection{Indirect Identification}

In general, in order that a direct identification approach yields consistent estimates using data collected in closed loop, it is necessary that the noise model $H(q, \theta)$ be of sufficient richness to encompass any true underlying noise colouring $H(q)$ [1]. This implies that arbitrary pre-filtering, which is equivalent to an arbitrary fixed noise model, cannot be employed in order to shape any suspected bias distribution.

In reaction to this, a range of so-called 'indirect' and 'joint input-output' identification methods have been developed in which the key idea is to first identify one or more transfer functions that form an open-loop model for known signals, and then in a subsequent step infer the required dynamics model from these initial estimates $[1,8,4,13]$.

The remainder of this paper is devoted to analysing the accuracy of these methods in comparison with one another, and in comparison to the accuracy of the direct identification method analysed in Theorem 4.1. We begin with a study of indirect methods, in which the unifying feature is the consideration of the relationship (3) to suggest the use of the model structure

$$
y_{t}=T(q, \theta) r_{t}+S H(q, \theta) e_{t} .
$$

The rationale for this choice is that since $\left\{e_{t}\right\}$ is typically uncorrelated with $\left\{r_{t}\right\}$, then the estimation of $T(q)$ on the basis of the observations $\left\{y_{t}\right\},\left\{r_{t}\right\}$ can be achieved with a fixed noise model/prefilter while still resulting in a consistent estimate. However, in what follows, it will be assumed that $\widehat{\theta}_{N}$ is formed according to (16) with associated prediction error

$$
\varepsilon_{t}(\theta)=S H^{-1}(q, \theta)\left[y_{t}-T(q, \theta) r_{t}\right] .
$$

The estimate $T\left(q, \widehat{\theta}_{N}\right)$ can then be used to derive an 'indirect' estimate $G\left(q, \widehat{\theta}_{N}\right)$ according to (5) as

$$
G\left(q, \widehat{\theta}_{N}\right)=\frac{T\left(q, \widehat{\theta}_{N}\right)}{K(q)\left[1-T\left(q, \widehat{\theta}_{N}\right)\right]} .
$$


Therefore, aside from the advantages attendant to being an open loop scenario, the indirect approach suffers from the need to know the controller $K(q)$.

With these essential features in mind, the various indirect estimation approaches then differ in their choice of parameterisation for $T(q, \theta)$, as will now be made explicit.

\subsubsection{Basic Indirect Identification}

What will here be called 'basic' indirect identification involves the choice of the following obvious choice of model structure

$$
T(q, \theta)=\frac{\beta_{0}+\beta_{1} q^{-1}+\cdots+\beta_{m_{\beta}} q^{-m_{\beta}}}{1+\alpha_{1} q^{-1}+\cdots+\alpha_{m_{\alpha}} q^{-m_{\alpha}}}
$$

and $S H(q, \theta)$ is parameterised independently of $T(q, \theta)$ and in such a manner to encompass the true underlying $S(q) H(q)$.

It is natural to also question the likely accuracy of $G\left(e^{j \omega}, \widehat{\theta}_{N}\right)$ found via (32) in relation to that of a direct approach, where in that case the accuracy has been quantified by (18). This question has been addressed in [1] where it is observed that according to the existing quantification (18)

$$
\operatorname{Var}\left\{T\left(e^{j \omega}, \widehat{\theta}_{N}\right)\right\} \approx \frac{m}{N} \frac{\left|S\left(e^{j \omega}\right)\right|^{2} \Phi_{\nu}(\omega)}{\Phi_{r}(\omega)}
$$

Actually, in the above, $m$ should be the order of $T\left(q, \widehat{\theta}_{N}\right)$ which, except for the case of proportional control, will be higher than that of the underlying model $G\left(q, \widehat{\theta}_{N}\right)$. We will ignore this point for the sake of the present discussion. In this case, since

$$
\mathrm{d} G=\frac{\mathrm{d} G}{\mathrm{~d} T} \mathrm{~d} T=\frac{1}{K S^{2}} \mathrm{~d} T
$$

then the arguments in $[1,4]$ indicate that the accuracy of $G\left(e^{j \omega}, \widehat{\theta}_{N}\right)$ found via this indirect approach can be quantified as [6]

$$
\operatorname{Var}\left\{G\left(e^{j \omega}, \widehat{\theta}_{N}\right)\right\} \approx \frac{m}{N} \frac{\left|S\left(e^{j \omega}\right)\right|^{2} \Phi_{\nu}(\omega)}{\Phi_{r}(\omega)} \frac{1}{\left|K\left(e^{j \omega}\right) S^{2}\left(e^{j \omega}\right)\right|^{2}}=\frac{m}{N} \frac{\Phi_{\nu}(\omega)}{\Phi_{u}^{r}(\omega)} .
$$

Using this method, the work in $[1,4]$ then concludes that, under an assumption of large (tending to infinity) model order $m$, the likely accuracy (as quantified by expressions such as (35)) for various indirect approaches is identical to that of (18) for a direct approach.

The first main result in the context of indirect identification methods employs Theorem 3.1 to examine a different by related question of frequency domain accuracy for finite, and possibly small model order.

Theorem 4.2. Suppose that the Standing Assumptions 2.1 are satisfied. Suppose further that the polynomial $A_{c}(z) \widetilde{A}(z) L(z)$ is of order less than or equal to the sum of the numerator and denominator model orders $m_{\alpha}+m_{\beta}$. Then in the case of the indirect identification procedure (30)- (32)

$$
\lim _{N \rightarrow \infty} N \operatorname{Var}\left\{G_{\text {id }}\left(e^{j \omega}, \widehat{\theta}_{N}\right)\right\}=\frac{\sigma^{2}}{\Phi_{u}(\omega)} \cdot \kappa_{\mathrm{id}}(\omega)
$$

where $\kappa_{\mathrm{id}}(\omega)$ is given by (20) with the associated zeros $\left\{\xi_{1}, \cdots, \xi_{m_{a}+m_{b}}\right\}$ being defined as those of the polynomial $z^{m_{\alpha}+m_{\beta}} A_{c}(z) \widetilde{A}(z) L(z)$. 
Proof. See Appendix A.2.

As per the case of the previous Theorem 4.1, this result suggests a variance quantification whose accuracy depends only on $N$ being sufficiently large, and in that sense is exact for finite model order:

$$
\operatorname{Var}\left\{G_{\mathrm{id}}\left(e^{j \omega}, \widehat{\theta}_{N}\right)\right\} \approx \frac{\sigma^{2}}{N \Phi_{u}^{r}(\omega)} \kappa_{\mathrm{id}}(\omega)
$$

There are several important conclusions to be drawn from this quantifications and the associated one (24) pertaining to direct identification.

1. First, and most important, they indicate that from an estimation accuracy point of view, direct and indirect identification are not equivalent. Both quantifications (37) and (24) depend on the closed loop poles defined by $A_{c}(z)$.

However, according to (37) the indirect method estimation error $\operatorname{Var}\left\{G_{\text {id }}\left(e^{j \omega}, \widehat{\theta}_{N}\right)\right\}$ also depends on a subset of the open loop poles defined by $\widetilde{A}(z)$ and the controller poles defined by $L(z)$. On the other hand (24) indicates that, modulo effects on $\Phi_{u}(\omega)$, the direct method estimation error $\operatorname{Var}\left\{G_{\mathrm{di}}\left(e^{j \omega}, \widehat{\theta}_{N}\right)\right\}$ does not depend on these poles, but does depend on all the open loop poles of the system $G(q)$.

Of course, it is entirely possible for the controller poles defined and open loop system poles to be very different and hence for the estimation errors to be very different. This will shortly be demonstrated via simulation example.

However, one situation in which the controller poles and open loop poles are the same is if the controller (10) is in fact simply a proportional controller $K(q)=K$ for some constant $K$, and in this case when $\mu \gg \sigma^{2}$ so that $\Phi_{u}^{r}(\omega) \approx \Phi_{u}(\omega)$ then $\operatorname{Var}\left\{G_{\mathrm{id}}\left(e^{j \omega}, \widehat{\theta}_{N}\right)\right\} \approx \operatorname{Var}\left\{G_{\mathrm{di}}\left(e^{j \omega}, \widehat{\theta}_{N}\right)\right\}$.

2. This difference between the variability of finite model order frequency response estimates obtained by direct and indirect methods is consistent with previous work. In particular, this difference is established in parameter space in [1]. The novel contribution here is to provide a frequency domain analysis that is also valid for finite model order.

3. In comparing direct and indirect methods by comparing $\kappa_{\mathrm{di}}(\omega)$ and $\kappa_{\mathrm{id}}(\omega)$, note that both these terms will be of the generic form

$$
\kappa(\omega)=m_{\star}+\sum_{k=1}^{2 m-m_{\star}} \frac{1-\left|\xi_{k}\right|^{2}}{\left|e^{j \omega}-\xi_{k}\right|^{2}}
$$

where $m$ is the McMillan degree of the true underlying system, and $m_{\star}$ is the number of numerator lags in the model structure that are excess of this degree, while the $\left\{\xi_{k}\right\}$ are the zeros of $A_{c}(z) A(z)$ or $A_{c}(z) \widetilde{A}(z) L(z)$ as appropriate.

For cases of large model order with many lags in the numerator, the constant $m_{\star}$ will dominate (38) and hence $\kappa_{\mathrm{di}}(\omega)$ and $\kappa_{\mathrm{id}}(\omega)$ will be essentially equivalent.

On the other hand, while (38) illustrates that the expressions (37) and (24) both imply (in the high SNR case where $\Phi_{u} \approx \Phi_{u}^{r}$ ) the asymptotic result

$$
\lim _{m_{\star} \rightarrow \infty} \lim _{N \rightarrow \infty} \frac{N}{m_{\star}} \operatorname{Var}\left\{G\left(e^{j \omega}, \widehat{\theta}_{N}\right)\right\}=\frac{\sigma^{2}}{\Phi_{u}(\omega)}
$$


it should be clear from the afore-mentioned potential differences between (24) and (37) that there can be serious pitfalls in concluding the approximate quantification

$$
\operatorname{Var}\left\{G\left(e^{j \omega}, \widehat{\theta}_{N}\right)\right\} \approx \frac{m_{\star}}{N} \frac{\sigma^{2}}{\Phi_{u}(\omega)}
$$

on the basis of the asymptotic result (39).

4. Note that there is a more subtle difference between the accuracy of direct and indirect estimates in that the quantification (24) shows that the variance of an indirect estimate depends only on that part of the input spectrum $\Phi_{u}^{r}(\omega)$ deriving from the external reference $\left\{r_{t}\right\}$, while the variance of a direct estimate depends on the total input power $\Phi_{u}(\omega)>\Phi_{u}^{r}(\omega)$. This has been previously observed and analysed in [1] using an asymptotic in model order argument. It is mentioned again here simply to to underline that it is also consistent with the finite model order analysis pursued in this paper.

\subsubsection{Identification via Tailor Made Parameterisation}

An alternative to the parameterisation (33) is the so-called 'tailor made' one [1] in which, (30) is again employed, and according to (5), the model structure

$$
T(q, \theta)=\frac{K(q) G(q, \theta)}{1+K(q) G(q, \theta)}, \quad G(q, \theta)=\frac{B(q, \theta)}{A(q, \theta)}
$$

is used with $A(q, \theta)$ and $B(q, \theta)$ being given by (12), (13) and $\theta$ containing the parameterisation of these polynomials, together with the independent parameterisation of the noise model $S H(q, \theta)$ in (30).

The advantage of this parameterisation is that it avoids the 'inversion' step (32), and in so doing guarantees the orders $m_{a}, m_{b}$ for the final estimated $G\left(q, \widehat{\theta}_{N}\right)$ which, as before, is found by minimisation of the prediction error residual (31).

Theorem 4.3. Suppose that the Standing Assumptions 2.1 are satisfied. Suppose further that the polynomial $A_{c}(z) A(z)$ is of order less than or equal to the sum of the numerator and denominator model orders $m_{a}+m_{b}$. Then in the case of identification with tailor made parameterisation (30), (41)

$$
\lim _{N \rightarrow \infty} N \operatorname{Var}\left\{G_{\mathrm{ta}}\left(e^{j \omega}, \widehat{\theta}_{N}\right)\right\}=\frac{\sigma^{2}}{\Phi_{u}^{r}(\omega)} \cdot \kappa_{\mathrm{di}}(\omega)
$$

where $\kappa_{\mathrm{di}}(\omega)$ is that specified in previously in Theorem 4.1 in relation to a direct estimation approach.

Proof. See Appendix A.3.

Comparing (42) with (23) then indicates that, since the function $\kappa_{\mathrm{di}}(\omega)$ defined by the zeros of $z^{m_{a}+m_{b}} A_{c}(z) A(z)$ occurs in both expressions, then the only difference between the accuracy of direct estimates, and indirect ones that utilise the tailor made parameterisation (41) is via the difference between the input spectrum $\Phi_{u}(\omega)$ and the spectrum $\Phi_{u}^{r}(\omega)$ of that component of the input $\left\{u_{t}\right\}$ that is derived from $\left\{r_{t}\right\}$. Since according to (6) and (7) the differences between these spectra become smaller as the $\left\{r_{t}\right\}$ to $\left\{e_{t}\right\}$ SNR increases, this further implies approximately equivalent relative accuracy between direct estimates, and indirect ones achieved by the tailor made parameterisation (41). 


\subsubsection{Identification via Dual-Youla Parameterisation}

The final indirect estimation method to be considered here is one based on the so-called dual-Youla parameterisation of all systems stabilised by a given controller. Namely, suppose that the controller $K(q)$, and a nominal, user chosen system $G^{0}(q)$ which is stabilised by $K(q)$ have co-prime representation [11] with respect to stable $M(q), N(q), X(q), Y(q)$ as

$$
G^{0}(q)=N(q) M^{-1}(q), \quad K(q)=X(q) Y^{-1}(q) .
$$

Then a further system $G(q, \theta)$, parameterised by $\theta \in \mathbf{R}^{n}$, is also stabilised by $K(q)$ if and only if there exists a stable and proper $R(q, \theta)$ such that [11]

$$
G(q, \theta)=\frac{N(q)+Y(q) R(q, \theta)}{M(q)-X(q) R(q, \theta)}
$$

Substituting this parameterisation into (5) then implies a parameterisation of $T(q, \theta)$ in terms of $R(q, \theta)$ according to

$$
T(q, \theta)=T^{0}(q)+M^{-1}(q) X(q) S^{0}(q) R(q, \theta)
$$

where $T^{0}(q)$ and $S^{0}(q)$ arise from (5) with the substitution $G(q)=G^{0}(q)$. Further substitution of (45) into (30) then leads to

$$
z_{t}=R(q, \theta) x_{t}+W(q, \theta) e_{t}
$$

where

$$
z_{t} \triangleq y_{t}-T^{0}(q) r_{t}, \quad x_{t}=M^{-1}(q) X(q) S^{0}(q) r_{t}, \quad W(q, \theta)=S H(q, \theta) .
$$

Here, if $\left\{r_{t}\right\}$ and $\left\{e_{t}\right\}$ are uncorrelated, then so are $\left\{x_{t}\right\}$ and $\left\{e_{t}\right\}$ and hence finding an estimate $\widehat{\theta}_{N}$ via (16) and the prediction error residual suggested by (46) of

$$
\varepsilon_{t}(\theta)=S H^{-1}(q, \theta)\left[z_{t}-R(q, \theta) x_{t}\right]
$$

is an open loop estimation problem $[11,1,13]$. Furthermore, it is essentially a re-parameterisation of the indirect estimation model structure (30) in which an estimate $G_{\mathrm{dy}}(q, \widehat{\theta})$ may be derived from $R\left(q, \widehat{\theta}_{N}\right)$ via (44). An attractive feature of this re-parameterisation is that the estimated $G_{\mathrm{dy}}\left(q, \widehat{\theta}_{N}\right)$ is guaranteed to be stabilised by the given controller $K(q)$.

Note further that an alternative and more commonly presented version of this parameterisation is one in which both sides of (46) are pre-filtered by $Y^{-1}(q)[11,1,13,4]$. However, this pre-filtering has no effect on the final variance of the estimate $G_{\mathrm{dy}}\left(q, \widehat{\theta}_{N}\right)$, and hence this paper will not consider it further.

In order to examine the accuracy of $G_{\mathrm{dy}}\left(q, \widehat{\theta}_{N}\right)$, it will be expedient to restrict attention to the following class of co-prime factorisations

$$
N(q)=\frac{B^{0}(q)}{E_{G}(q)}, \quad M(q)=\frac{A^{0}(q)}{E_{G}(q)}, \quad X(q)=K \frac{P(q)}{E_{K}(q)}, \quad Y(q)=\frac{L(q)}{E_{K}(q)}
$$

where in $X(q), K \in \mathbf{R}$ and all the terms on the right hand side of equals signs in (47) are polynomial in $q^{-1}, E_{G}(q)$ and $E_{K}(q)$ are user-chosen Schur polynomials, and $G^{0}(q)=B^{0}(q) / A^{0}(q), K(q)=$ $P(q) / L(q)$. Furthermore, it will be assumed that the model structure $R(q, \theta)$ is of the form

$$
R(q, \theta)=\frac{\lambda^{0}+\lambda_{1} q^{-1}+\cdots+\lambda_{m_{\lambda}} q^{-m_{\lambda}}}{1+\gamma_{1} q^{-1}+\cdots+\gamma_{m_{\gamma}} q^{-m_{\gamma}}}
$$


and that $W(q, \theta)$ is parameterised independently of $R(q, \theta)$, and is of sufficient order to encompass $Y^{-1}(q) S(q) H(q)$. With this in mind, the following theorem provides the main result of this subsection.

Theorem 4.4. Suppose that the Standing Assumptions 2.1 are satisfied. Suppose further that the polynomial $E_{G}(z) E_{K}(z) P(z) A_{c}(z) A_{c}^{0}(z)$ is of order less than or equal to the sum of the numerator and denominator model orders $m_{\gamma}+m_{\lambda}$, where $A_{c}(z)$ was defined in (27) and

$$
A_{c}^{0}(z) \triangleq A^{0}(z) L(z)+K P(z) B^{0}(z) .
$$

Then in the case of indirect identification using the dual-Youla parameterisation (43)-(48)

$$
\lim _{N \rightarrow \infty} N \operatorname{Var}\left\{G_{\text {dy }}\left(e^{j \omega}, \widehat{\theta}_{N}\right)\right\}=\frac{\sigma^{2}}{\Phi_{u}^{r}(\omega)} \kappa_{\text {dy }}(\omega)
$$

where $\kappa_{\mathrm{dy}}(\omega)$ is as defined in (20) with the associated zeros $\left\{\xi_{1}, \cdots, \xi_{m_{\lambda}+m_{\gamma}}\right\}$ being those of

$$
z^{m_{\gamma}+m_{\lambda}} E_{G}(z) E_{K}(z) A(z) A_{c}(z) A_{c}^{0}(z) .
$$

Proof. See Appendix A.4.

As in previous cases, this new result invites comment.

1. Firstly, and most obviously, the associated quantification, exact for finite model order, of

$$
\operatorname{Var}\left\{G_{\mathrm{dy}}\left(e^{j \omega}, \widehat{\theta}_{N}\right)\right\} \approx \frac{\sigma^{2}}{N \Phi_{u}^{r}(\omega)} \kappa_{\mathrm{dy}}(\omega)
$$

and compared to (37), (24) and (55), indicates that in general, the accuracy of estimates $G_{\mathrm{dy}}\left(q, \widehat{\theta}_{N}\right)$ obtained by the dual Youla method are not equivalent to those obtained by direct, basic indirect, or co-prime factor methods. This is due to the dependence of (50) on the zeros of the nominal closed loop denominator $A_{c}^{0}(q)$ defined in (A.2), and on the zeros of $E_{G}(z)$ and $E_{K}(z)$ used in the definition of the co-prime factorisations of $G^{0}(z)$ and $K(z)$. Since $A_{c}^{0}(q), E_{G}(z)$ and $E_{K}(z)$ can be rather arbitrary (modulo the requirement they be Schur), then $\operatorname{Var}\left\{G_{\text {dy }}\left(e^{j \omega}, \widehat{\theta}_{N}\right)\right\}$ can also be very different to the variance of other indirect, and also direct system estimates.

Indeed, for $\mu \gg \sigma^{2}$ so that $\Phi_{u}^{r}(\omega) \approx \Phi_{u}(\omega)$ then comparison with (24) indicates that with the choices $E_{K}(z)=E_{G}(z)=1$ and with $\left\{\rho_{k}\right\}$ being the zeros of $A_{c}^{0}(z)$, and $K(q)$ being a pole cancelling design with $P(q)=K A(q)$, then

$$
\operatorname{Var}\left\{G_{\mathrm{dy}}\left(e^{j \omega}, \widehat{\theta}_{N}\right)\right\} \approx \operatorname{Var}\left\{G_{\mathrm{di}}\left(e^{j \omega}, \widehat{\theta}_{N}\right)\right\}+\frac{\sigma^{2}}{N \Phi_{u}^{r}(\omega)} \sum_{k=1}^{m_{\rho}} \frac{1-\left|\rho_{k}\right|^{2}}{\left|e^{j \omega}-\rho_{k}\right|^{2}}
$$

and hence, under the conditions of the preceding theorems, it could be expected that regardless of the choice of $G^{0}(q)$, the accuracy of the dual Youla method will be inferior to that of a direct method.

2. In the preceding cases, it was illustrated that in the special case of proportional control $K(q)=$ $K q^{-1}$, the variance of the basis indirect and co-prime factor methods became equal to that of the direct method. Such is not the case for the dual-Youla approach. Regardless of the controller being proportional, there is a further degree of freedom in the dual-Youla approach, namely the choice of nominal system $G^{0}(q)$, which introduces nominal closed loop poles $\left\{\rho_{k}\right\}$ into the variance quantification (51) and hence precludes $\operatorname{Var}\left\{G_{\mathrm{dy}}\left(e^{j \omega}, \widehat{\theta}_{N}\right)\right\}$ from ever being equal to $\operatorname{Var}\left\{G_{\mathrm{di}}\left(e^{j \omega}, \widehat{\theta}_{N}\right)\right\}$, regardless of the choice of controller. 
3. Although, in the interests of most clearly exposing certain principles, the particular co-prime factorisation (47) was chosen, it is also possible to handle more general cases using the same method as in the proof of Theorem 4.4, but possibly at the expense of more cumbersome notation.

\subsection{Joint Input-Output Identification}

The final closed loop identification strategy to be studied here is the class of so-called 'joint inputoutput' methods, in which two model structures

$$
\begin{aligned}
& y_{t}=T(q, \theta) r_{t}+S H(q, \theta) e_{t} \\
& u_{t}=S K(q, \beta) r_{t}-S K H(q, \beta) e_{t}
\end{aligned}
$$

are used. These structures imply the following one-step ahead prediction errors

$$
\begin{aligned}
\varepsilon_{t}^{y}(\theta) & =S H^{-1}(q, \theta)\left[y_{t}-T(q, \theta) r_{t}\right] \\
\varepsilon_{t}^{u}(\beta) & =S K H^{-1}(q, \beta)\left[u_{t}-S K(q, \beta) r_{t}\right]
\end{aligned}
$$

which are used to find estimates $\widehat{\theta}_{N}, \widehat{\beta}_{N}$ according to

$$
\left[\widehat{\theta}_{N}, \widehat{\beta}_{N}\right]=\underset{\theta, \beta}{\arg \min } V_{N}(\theta, \beta), \quad V_{N}(\theta, \beta) \triangleq \frac{1}{2 N} \sum_{t=1}^{N}\left[\varepsilon_{t}^{y}(\theta)\right]^{2}+\left[\varepsilon_{t}^{u}(\beta)\right]^{2} .
$$

Since $\left\{r_{t}\right\}$ is not correlated with $\left\{e_{t}\right\}$, then this implies the equivalent of two open loop estimation problems, with all the attendant advantages of this scenario as mentioned earlier. The estimate of the open loop dynamics is then found via this joint input-output method as

$$
G_{\mathrm{jio}}\left(q, \widehat{\theta}_{N}, \widehat{\beta}_{N}\right)=\frac{T\left(q, \widehat{\theta}_{N}\right)}{S K\left(q, \widehat{\beta}_{N}\right)} .
$$

Note that an advantage of this method in comparison to the indirect methods just studied is that knowledge of the controller $K(q)$ is not required. Furthermore, there exists a range of possible joint input-output methods that depend on the model structures used in (52), (53). In this paper, two are considered, the 'basic' joint input-output method and the 'co-prime factor' method.

\subsubsection{Basic Joint Input-Output Identification}

The so-called 'basic' joint input-output approach involves using a model structure for $T(q, \theta)$ given by (33) and for $S K(q, \beta)$ given by

$$
S K(q, \beta)=\frac{\rho_{0}+\rho_{1} q^{-1}+\cdots+\rho_{m_{\rho}} q^{-m_{\rho}}}{1+\delta_{1} q^{-1}+\cdots+\delta_{m_{\delta}} q^{-m_{\delta}}} .
$$

The model structures for the noise models $S H(q, \theta)$ and $S K H(q, \beta)$ are assumed to be independently parameterised from the dynamics models $T(q, \theta), S K(q, \beta)$, and such that they can completely describe the true noise models $S(q) H(q), S(q) K(q) H(q)$ without any pole-zero cancellations.

The variance properties of the resulting basic joint input-output estimate $G_{\text {jio }}\left(q, \widehat{\theta}_{N}, \widehat{\beta}_{N}\right)$ derived from (55), and under the same conditions considered in the previous sections, are now established via the following theorem. 
Theorem 4.5. Suppose that the Standing Assumptions 2.1 are satisfied. Suppose further that the model orders chosen for $T(q, \theta)$ satisfies the conditions of Theorem 4.2 so that $A_{c}(z) \widetilde{A}(z) L(z)$ is a polynomial in $z^{-1}$ of order less than $m_{\alpha}+m_{\beta}$. Finally, suppose that the model orders chosen for $S K(q, \beta)$ are such that $A_{c}(z) A(z)$ is a polynomial in $z^{-1}$ of order less than $m_{\delta}+m_{\rho}$. Then using the joint input-output identification method, it holds that

$$
\begin{aligned}
\lim _{N \rightarrow \infty} N \operatorname{Var}\left\{G_{\mathrm{jio}}\left(e^{j \omega}, \widehat{\theta}_{N}, \widehat{\beta}_{N}\right)\right\}= & \left|S\left(e^{j \omega}\right)\right|^{2} \lim _{N \rightarrow \infty} N \operatorname{Var}\left\{G_{\mathrm{id}}\left(e^{j \omega}, \widehat{\theta}_{N}\right)\right\}+ \\
& \left(\frac{\mu}{\mu+\sigma^{2}}\right)\left|T\left(e^{j \omega}\right)\right|^{2} \lim _{N \rightarrow \infty} N \operatorname{Var}\left\{G_{\mathrm{di}}\left(e^{j \omega}, \widehat{\theta}_{N}\right)\right\}+ \\
& \frac{2 \sigma^{2}}{\Phi_{u}^{r}(\omega)} \operatorname{Re}\left\{T\left(e^{j \omega}\right) \overline{S\left(e^{j \omega}\right)} \Delta(\omega)\right\}
\end{aligned}
$$

where $\Delta(\omega)$ is specified in the following equation (58) and

$$
\lim _{N \rightarrow \infty} N \operatorname{Var}\left\{G_{\mathrm{di}}\left(e^{j \omega}, \widehat{\theta}_{N}\right)\right\}, \quad \lim _{N \rightarrow \infty} N \operatorname{Var}\left\{G_{\mathrm{id}}\left(e^{j \omega}, \widehat{\theta}_{N}\right)\right\}
$$

are given by (36) with $m_{\alpha}, m_{\beta}$ the same as here, and (23) with $m_{a}=m_{\delta}, m_{b}=m_{\rho}$.

Proof. See Appendix A.5.

As per the previous theorem, this result suggests the following approximate quantification which is 'exact' for finite model order, but is of increasing accuracy with increasing data length $N$

$$
\begin{aligned}
\operatorname{Cov}\left\{G_{\mathrm{co}}\left(e^{j \omega}, \widehat{\theta}_{N}, \widehat{\beta}_{N}\right)\right\} \approx & \left|S\left(e^{j \omega}\right)\right|^{2} \operatorname{Var}\left\{G_{\mathrm{id}}\left(e^{j \omega}, \widehat{\theta}_{N}\right)\right\}+\left(\frac{\mu+\sigma^{2}}{\mu}\right)\left|T\left(e^{j \omega}\right)\right|^{2}\left\{G_{\mathrm{di}}\left(e^{j \omega}, \widehat{\theta}_{N}\right)\right\} \\
& +\frac{2}{N} \frac{\sigma^{2}}{\Phi_{u}^{r}(\omega)} \operatorname{Re}\left\{T\left(e^{j \omega}\right) \overline{S\left(e^{j \omega}\right)} \Delta(\omega)\right\} .
\end{aligned}
$$

Some comments about this result are clearly in order.

1. Firstly, the quantification (57) clearly shows that, just as the previous section has established that the variance $\operatorname{Var}\left\{G_{\text {id }}\left(e^{j \omega}, \widehat{\theta}_{N}\right)\right\}$ for the basic indirect method estimate is not, in general, the same as the variance $\operatorname{Var}\left\{G_{\mathrm{di}}\left(e^{j \omega}, \widehat{\theta}_{N}\right)\right\}$ for a direct methods estimate, the new quantification (56) shows that the variance $\operatorname{Var}\left\{G_{\text {jio }}\left(e^{j \omega}, \widehat{\theta}_{N}, \widehat{\beta}_{N}\right)\right\}$ for basic joint input-output methods is also not, in general, equal to either of these.

In fact, consideration of only the first two terms in (57) illustrates that under the assumptions of Theorem $4.5, \operatorname{Var}\left\{G_{\mathrm{co}}\left(e^{j \omega}, \widehat{\theta}_{N}, \widehat{\beta}_{N}\right)\right\}$ is a pseudo-convex combination of $\operatorname{Var}\left\{G_{\text {id }}\left(e^{j \omega}, \widehat{\theta}_{N}\right)\right\}$ and $\operatorname{Var}\left\{G_{\mathrm{di}}\left(e^{j \omega}, \widehat{\theta}_{N}\right)\right\}$, where the epithet 'pseudo' is used since $S+T=1$ and hence if $\mu \gg \sigma^{2}$ then

$$
\left|S\left(e^{j \omega}\right)\right|^{2}+\left(\frac{\mu+\sigma^{2}}{\mu}\right)\left|T\left(e^{j \omega}\right)\right|^{2} \approx 1 .
$$

Therefore, again ignoring the last $\Delta(\omega)$ term of (57) shows that while at some frequencies where $|S| \approx 1$ or $|T| \approx 1$ then the variance of joint input-output methods estimates might be approximately the same as either a direct method or basic indirect method estimate, it is certainly not in general equal to either of them. 
2. Turning now to the last component of (57), the term $\Delta(\omega)$ needs to be defined. In fact it is given as

$$
\Delta(\omega) \triangleq \frac{1}{2 \pi} \int_{-\pi}^{\pi} \Delta_{1}(\lambda, \omega) \overline{\Delta_{2}(\lambda, \omega)} \mathrm{d} \lambda
$$

where

$$
\Delta_{1}(\lambda, \omega)=\sum_{k=1}^{m_{\alpha}+m_{\beta}} \mathcal{B}_{k}\left(e^{j \lambda}\right) \overline{\mathcal{B}_{k}\left(e^{j \omega}\right)}, \quad \Delta_{2}(\lambda, \omega)=\sum_{k=1}^{m_{\delta}+m_{\rho}} \mathcal{F}_{k}\left(e^{j \lambda}\right) \overline{\mathcal{F}_{k}\left(e^{j \omega}\right)},
$$

and with $\left\{\zeta_{k}\right\}$ being the zeros of $z^{m_{\alpha}+m_{\beta}} A_{c}(z) \widetilde{A}(z) L(z)$ and $\left\{\tau_{k}\right\}$ being the zeros of $z^{m_{\delta}+m_{\rho}} A_{c}(z) A(z)$

$$
\mathcal{B}_{k}(z) \triangleq \frac{z \sqrt{1-\left|\zeta_{k}\right|^{2}}}{z-\zeta_{k}} \phi_{k-1}(z), \quad \phi_{k}(z) \triangleq \prod_{\ell=1}^{k} \frac{1-\overline{\zeta_{\ell}} z}{z-\zeta_{\ell}}, \quad \phi_{0}(z) \triangleq 1
$$

while

$$
\mathcal{F}_{k}(z) \triangleq \frac{z \sqrt{1-\left|\tau_{k}\right|^{2}}}{z-\tau_{k}} \psi_{k-1}(z), \quad \psi_{k}(z) \triangleq \prod_{\ell=1}^{k} \frac{1-\overline{\tau_{\ell}} z}{z-\tau_{\ell}}, \quad \psi_{0}(z) \triangleq 1 .
$$

Although this definition of $\Delta(\omega)$ appears quite complicated, it has a simple geometric interpretation, that is obtained by considering the spaces

$$
\begin{gathered}
V_{\zeta} \triangleq \operatorname{Span}\left\{\frac{1}{\prod_{k=1}^{m_{\alpha}+m_{\beta}}\left(1-\zeta_{k} z^{-1}\right)}, \cdots, \frac{\left.z^{-\left(m_{\alpha}+m_{\beta}\right)}\right)}{\prod_{k=1}^{m_{\alpha}+m_{\beta}}\left(1-\zeta_{k} z^{-1}\right)}\right\} \\
V_{\tau} \triangleq \operatorname{Span}\left\{\frac{1}{\prod_{k=1}^{m_{\delta}+m_{\rho}}\left(1-\tau_{k} z^{-1}\right)}, \cdots, \frac{\left.z^{-\left(m_{\delta}+m_{\rho}\right)}\right)}{\prod_{k=1}^{m_{\delta}+m_{\rho}}\left(1-\tau_{k} z^{-1}\right)}\right\} .
\end{gathered}
$$

With this in mind, define $P_{V_{\zeta}}: H_{2} \rightarrow V_{\zeta}$ as the orthogonal projection of an arbitrary function $f \in H_{2}$ onto $V_{\zeta}$ :

$$
P_{V_{\zeta}} f \mapsto \widehat{f} \in V_{\zeta} \text { such that }\langle\widehat{f}-f, g\rangle=0 \forall g \in V_{\zeta},
$$

and similarly $P_{V_{\tau}}: H_{2} \rightarrow V_{\tau}$ is the orthogonal projection onto $V_{\tau}$. Then these projections can be given an explicit formulation via $\Delta_{1}(\lambda, \omega)$ and $\Delta_{2}(\lambda, \omega)$ as [10]

$$
\left[P_{V_{\zeta}} f\right](\omega)=\frac{1}{2 \pi} \int_{-\pi}^{\pi} f(\lambda) \overline{\Delta_{1}(\lambda, \omega)} \mathrm{d} \lambda, \quad\left[P_{V_{\tau}} f\right](\omega)=\frac{1}{2 \pi} \int_{-\pi}^{\pi} f(\lambda) \overline{\Delta_{2}(\lambda, \omega)} \mathrm{d} \lambda .
$$

Therefore, according to (58), $\Delta(\omega)$ is the best (in the sense of minimal $H_{2}$ norm of error) approximation of $\Delta_{1}(\lambda, \omega)$ (which, as a function of $\lambda$ is in $V_{\zeta}$ for all $\omega$ ) in terms of an element $f \in V_{\tau}$.

With this in mind, an explicit expression for $\Delta(\omega)$ arises by noting that $\left\{\mathcal{F}_{k}\right\}$ is an orthonormal basis for $V_{\tau}$ so that

$$
\Delta(\omega)=\sum_{n=1}^{m_{\delta}+m_{\rho}} \sum_{k=1}^{m_{\alpha}+m_{\beta}}\left\langle\mathcal{B}_{k}\left(e^{j \lambda}\right), \mathcal{F}_{n}\left(e^{j \lambda}\right)\right\rangle \overline{\mathcal{B}_{k}\left(e^{j \omega}\right)} \mathcal{F}_{n}\left(e^{j \omega}\right)
$$


This representation highlights that $\Delta(\omega)$ is a rational function that contains poles equal to the open and closed loop poles, and at the complex conjugate of the open loop poles and the controller poles. Unfortunately since, in general, the inner product term has quite a complicated (but computable) closed form expression, then a simple formulation of $\Delta(\omega)$ does not seem possible. However, since $\Delta(\omega)$ is the sum of terms that are of indefinite sign, while the first two terms in (56) involve sums of $\left|\mathcal{B}_{k}\left(e^{j \omega}\right)\right|^{2},\left|\mathcal{F}_{k}\left(e^{j \omega}\right)\right|^{2}$ which are all positive, then these first two terms could be expected to dominate the quantification, and it is the experience of the authors that this is generally true.

3. Consider the special case of proportional control in which $K(q)=K \in \mathbf{R}$ so that $\widetilde{A}(z)=A(z)$ and hence, provided that $\mu \gg \sigma^{2}$ so $\Phi_{u}^{r}(\omega) \approx \Phi_{u}^{r}(\omega)$ then

$$
\operatorname{Var}\left\{G_{\mathrm{id}}\left(e^{j \omega}, \widehat{\theta}_{N}\right)\right\}=\operatorname{Var}\left\{G_{\mathrm{di}}\left(e^{j \omega}, \widehat{\theta}_{N}\right)\right\} \approx \frac{\sigma^{2}}{N \Phi_{u}(\omega)} \kappa_{\mathrm{di}}(\omega) .
$$

In this same situation, the spaces $V_{\zeta}$ and $V_{\tau}$ in (60), (61) become the same, so that $\Delta_{2}(\lambda, \omega)=$ $\Delta_{1}(\lambda, \omega)$ and hence using the reproducing kernel property of $\Delta_{1}(\lambda, \omega)$

$$
\Delta(\omega)=\Delta_{1}(\omega, \omega)=\left[\left(m_{\beta}-m_{\alpha}\right)+\sum_{k=1}^{m_{\alpha}} \frac{1-\left|\eta_{k}\right|^{2}}{\left|e^{j \omega}-\eta_{k}\right|^{2}}+\sum_{k=1}^{m_{a}} \frac{1-\left|\xi_{k}\right|^{2}}{\left|e^{j \omega}-\xi_{k}\right|^{2}}\right]=\kappa_{\mathrm{di}}(\omega) .
$$

Consequently, substituting (62) and (63) into (57) indicates that in this special case of proportional control

$$
\begin{aligned}
\operatorname{Var}\left\{G_{\mathrm{co}}\left(e^{j \omega}, \widehat{\theta}_{N}, \widehat{\beta}_{N}\right)\right\} & =\operatorname{Var}\left\{G_{\mathrm{di}}\left(e^{j \omega}, \widehat{\theta}_{N}\right)\right\}\left[|S|^{2}+|T|^{2}+T \bar{S}\right] \\
& =\operatorname{Var}\left\{G_{\mathrm{di}}\left(e^{j \omega}, \widehat{\theta}_{N}\right)\right\}=\operatorname{Var}\left\{G_{\mathrm{id}}\left(e^{j \omega}, \widehat{\theta}_{N}\right)\right\} .
\end{aligned}
$$

While this last comment establishes that there may be situations, such as that of simple proportional control, in which direct and indirect methods deliver estimates of the same accuracy, the quantification (56) establishes that this is by no means the general situation.

\subsubsection{Coprime Factor Identification}

A generalisation of the basic joint input-output approach is the so-called 'co-prime factor method' [12, 11, 13], which starts from the relationships (52), (53) and introduces the filtered signal

$$
x_{t}=X(q) r_{t}, \quad X(q)=\frac{X_{N}(q)}{X_{D}(q)}
$$

where $X_{N}(q), X_{D}(q)$ are polynomials in $q^{-1}$ and which leads to

$$
\begin{aligned}
& y_{t}=N(q, \theta) x_{t}+S H(q, \theta) e_{t} \\
& u_{t}=M(q, \beta) x_{t}-S K H(q, \beta) e_{t}
\end{aligned}
$$

where

$$
N(q, \theta)=T(q, \theta) X^{-1}(q), \quad M(q, \beta)=S K(q, \beta) X^{-1}(q) .
$$

This is nothing more than a re-parameterisation of (52), (53) and suggests the use of the prediction error residuals

$$
\varepsilon_{t}^{y}(\theta)=S H^{-1}(q, \theta)\left[y_{t}-N(q, \theta) x_{t}\right], \quad \varepsilon_{t}^{u}(\beta)=S K H^{-1}(q, \beta)\left[u_{t}-M(q, \beta) x_{t}\right],
$$


as a method of obtaining estimates $\widehat{\theta}_{N}, \widehat{\beta}_{N}$ according to (54) which then delivers an estimate $G_{\mathrm{co}}\left(q, \widehat{\theta}_{N}, \widehat{\beta}_{N}\right)$ of the input-output dynamics according to

$$
G_{\mathrm{co}}\left(q, \widehat{\theta}_{N}, \widehat{\beta}_{N}\right)=\frac{N\left(q, \widehat{\theta}_{N}\right)}{M\left(q, \widehat{\beta}_{N}\right)} .
$$

The appended subscript co denotes that estimates obtained in this way have often been termed 'coprime factor method' estimates $[11,1,13,4]$ on account of the fact that it is possible to choose $X(q)$ such that $N\left(q, \widehat{\theta}_{N}\right), M(q, \widehat{\beta})$ are a normalised co-prime pair, in which case $G_{\mathrm{co}}\left(q, \widehat{\theta}_{N}, \widehat{\beta}_{N}\right)$ is of minimal order.

In order to study the accuracy of $G_{\mathrm{co}}\left(q, \widehat{\theta}_{N}, \widehat{\beta}_{N}\right)$, recall the 'generalised' direct estimate $G_{\mathrm{di} x}\left(q, \widehat{\theta}_{N}\right)$ defined in (25)-(26), which is a direct estimate using the same prefilter $X(q)$, and whose accuracy is quantified by (28), (29). Similarly, consider a generalisation of the preceding 'basic' indirect identification method in which the estimate $N\left(q, \widehat{\theta}_{N}\right)$ is obtained via the model structure (65) with

$$
N(q, \theta)=\frac{\beta_{0}+\beta_{1} q^{-1}+\cdots+\beta_{m_{\beta}} q^{-m_{\beta}}}{1+\alpha_{1} q^{-1}+\cdots+\alpha_{m_{\alpha}} q^{-m_{\alpha}}}
$$

and the prediction error residual (66) is used to form a 'generalised' indirect estimate $G_{\text {id } x}\left(q, \widehat{\theta}_{N}\right)$ of $G(q)$ according to

$$
G_{\mathrm{id} x}\left(q, \widehat{\theta}_{N}\right)=\frac{X(q) N\left(q, \widehat{\theta}_{N}\right)}{K(q)\left[1-X(q) N\left(q, \widehat{\theta}_{N}\right)\right]} .
$$

Therefore, since

$$
\mathrm{d} G_{\mathrm{id} x}=\frac{X}{K^{2} S} \mathrm{~d} N
$$

it follows using the arguments in the the proof of Theorem 4.6 that

$$
\lim _{N \rightarrow \infty} N \operatorname{Var}\left\{G_{\mathrm{id} x}\left(e^{j \omega}, \widehat{\theta}_{N}\right)\right\}=\frac{\sigma^{2}}{\Phi_{u}^{r}(\omega)} \kappa_{\mathrm{id} x}(\omega)
$$

where $\kappa_{\mathrm{id} x}(\omega)$ is defined according to (20) with the $\left\{\xi_{k}\right\}$ in that expression being the zeros of

$$
z^{m_{\alpha}+m_{\beta}} A_{c}(z) \widetilde{A}(z) L(z) X_{N}(z) X_{D}(z) .
$$

The purpose of considering these hypothetical estimates $\operatorname{Var}\left\{G_{\operatorname{di} x}\left(q, \widehat{\theta}_{N}\right)\right\}$ and $\operatorname{Var}\left\{G_{\mathrm{id} x}\left(q, \widehat{\theta}_{N}\right)\right\}$ together with their frequency domain variances given by (26), (68) is that they arise naturally in the quantification of the variance of system estimates formed via the co-prime factor method.

This is established in the following theorem, for which it is also necessary to specify that the model structure employed for estimation of $M(q)$ is of the form

$$
M(q, \beta)=\frac{\rho_{0}+\rho_{1} q^{-1}+\cdots+\rho_{m_{\rho}} q^{-m_{\rho}}}{1+\delta_{1} q^{-1}+\cdots+\delta_{m_{\delta}} q^{-m_{\delta}}} .
$$

With this in mind, the accuracy of the co-prime factor method of indirect identification may be quantified via the following result.

Theorem 4.6. Suppose that the Standing Assumption s2.1 are satisfied. Suppose further that the model orders chosen for $N(q, \theta)$ are such that that $X_{N}(z) X_{D}(z) A_{c}(z) \widetilde{A}(z) L(z)$ is a polynomial in $z^{-1}$ of order less than $m_{\alpha}+m_{\beta}$. Finally, suppose that the model orders chosen for $M(q, \beta)$ are such 
that $X_{N}(q) X_{D}(z) A_{c}(z) A(z)$ is a polynomial in $z^{-1}$ of order less than $m_{\delta}+m_{\rho}$. Then using the coprime factor identification method (64)-(67)

$$
\begin{aligned}
\lim _{N \rightarrow \infty} N \operatorname{Var}\left\{G_{\mathrm{co}}\left(e^{j \omega}, \widehat{\theta}_{N}, \widehat{\beta}_{N}\right)\right\}= & \left|S\left(e^{j \omega}\right)\right|^{2} \lim _{N \rightarrow \infty} N \operatorname{Var}\left\{G_{\mathrm{id} x}\left(e^{j \omega}, \widehat{\theta}_{N}\right)\right\}+ \\
& \left(\frac{\mu}{\mu+\sigma^{2}}\right)\left|T\left(e^{j \omega}\right)\right|^{2} \lim _{N \rightarrow \infty} N \operatorname{Var}\left\{G_{\mathrm{di} x}\left(e^{j \omega}, \widehat{\theta}_{N}\right)\right\}+ \\
& \frac{2 \sigma^{2}}{\Phi_{u}^{r}(\omega)} \operatorname{Re}\left\{T\left(e^{j \omega}\right) \overline{S\left(e^{j \omega}\right)} \Delta(\omega)\right\}
\end{aligned}
$$

where the asymptotic in $N$ values of $\operatorname{Var}\left\{G_{\mathrm{dix}}\left(e^{j \omega}, \widehat{\theta}_{N}\right)\right.$ and $\operatorname{Var}\left\{G_{\mathrm{id} x}\left(e^{j \omega}, \widehat{\theta}_{N}\right)\right.$ are given by $(28)$ and (69) respectively, and $\Delta(\omega)$ is as defined via (58)-(61) save that the spaces $V_{\tau}$ and $V_{\zeta}$ involved in that definition are both augmented to also include the poles and zeros of $X(z)$.

Proof. See Appendix A.6.

Again, via the suggested quantification

$$
\begin{aligned}
\operatorname{Var}\left\{G_{\mathrm{Co}}\left(e^{j \omega}, \widehat{\theta}_{N}, \widehat{\beta}_{N}\right)\right\} \approx & \frac{\left|S\left(e^{j \omega}\right)\right|^{2}}{N} \operatorname{Var}\left\{G_{\mathrm{id} x}\left(e^{j \omega}, \widehat{\theta}_{N}\right)\right\}+ \\
& \left(\frac{\mu}{\mu+\sigma^{2}}\right) \frac{\left|T\left(e^{j \omega}\right)\right|^{2}}{N} \operatorname{Var}\left\{G_{\mathrm{di} x}\left(e^{j \omega}, \widehat{\theta}_{N}\right)\right\}+ \\
& \frac{2 \sigma^{2}}{N \Phi_{u}^{r}(\omega)} \operatorname{Re}\left\{T\left(e^{j \omega}\right) \overline{S\left(e^{j \omega}\right)} \Delta(\omega)\right\}
\end{aligned}
$$

this result highlights that there can be appreciable differences in the accuracy of estimates obtained in closed loop, depending on the estimation strategy employed.

In particular, note that all the terms on the right hand side of the quantification (70) depend on both the poles and zeros of chosen pre-filter $X(q)$, which (of course) was not a factor in preceding quantifications for several other estimation methods.

Again, there is a special case worth mentioning of strictly proportional control, in which case (via an identical argument as used in the previous section), the quantification (70) reduces to

$$
\operatorname{Var}\left\{G_{\mathrm{co}}\left(e^{j \omega}, \widehat{\theta}_{N}, \widehat{\beta}_{N}\right)\right\}=\operatorname{Var}\left\{G_{\mathrm{di} x}\left(e^{j \omega}, \widehat{\theta}_{N}\right)\right\}=\operatorname{Var}\left\{G_{\mathrm{id} x}\left(e^{j \omega}, \widehat{\theta}_{N}\right)\right\} .
$$

However, again because of their dependence on the poles and zeros of $X(q)$, these latter two quantities are not equal to the variances for direct and basic indirect methods.

\section{Simulation Examples}

In order to emphasise the significance and ramifications of the new variance quantifications developed in this paper, this penultimate section provides a simulation example to illustrate the preceding results by considering the system

$$
y_{t}=G(q) u_{t}+e_{t}, \quad G(q)=\frac{1.6177 q^{2}-0.74 q}{q^{2}-1.8 q+0.81}
$$

which, according to (2) is under the influence of the pole cancelling controller

$$
K(q)=\frac{q^{2}-1.8 q+0.81}{q^{3}-1.9801 q^{2}+0.99 q}
$$


This choice of $K(q)$ implies $\widetilde{A}(z)=1$ according to (9) and delivers

$$
T(q)=\frac{1.618 q-0.74}{q^{2}-0.3624 q+0.25}, \quad S(q)=\frac{q^{2}-1.9801 q+0.99}{q^{2}-0.3624 q+0.25}
$$

and hence provides closed loop poles at $\eta_{1}, \eta_{2}=0.5 e^{ \pm j 1.2}$. While this might seem a relatively benign situation, note that the controller poles are at $\ell_{1}, \ell_{2}=0.995 e^{ \pm j 0.1}$, which are different to the open loop plant poles at $\xi_{1}, \xi_{2}=0.9$.

Therefore, according to (37) the estimation error involved with direct identification using a BoxJenkins model structure to first estimate $T(q)$ and $S(q)$, should be different to the estimation error given by (24) for the case of direct identification of $G(q)$ using an output-error structure. Indeed, as shown in Figure 2, simulation confirms this. In that figure the solid lines are the true variability as computed via Monte-Carlo simulation over 1000 estimate realisations, each derived from $N=1000$ data points with measurement noise variance $\sigma^{2}=10$ and input $\Phi_{r}(\omega)=1$. The solid line MonteCarlo variance with the large peak corresponds to the case of indirect identification, while the other solid line Monte-Carlo variance is for direct identification. Note that these actual mean square errors agree essentially perfectly with the quantifications (37) and (24) shown (respectively) as dashed and dash-dot lines; in the latter case, the new quantification (24) cannot be discerned from the sample variability solid line which exactly matches it and hence obliterates it.

Furthermore, note that as predicted by (37), the location of controller poles near the unit circle has a detrimental effect on indirect estimation accuracy at frequencies on the unit circle very close to those poles. In this case, the increased variability is at 0.1 radians/second which is near the plant crossover frequency, so that there are clear implications from a control design point of view as to whether direct or indirect estimation is preferred in this scenario.

By way of contrast, the lower (labelled) dash-dot line in Figure 2 is the existing quantification (18) which clearly provides a less informative quantification than (37), (24) and certainly does not expose the true accuracy differences between a direct and indirect approach.

To give a sense of the scale of estimation errors being quantified here, the first ten realisations of the estimate $G\left(e^{j \omega}, \widehat{\theta}_{N}\right)$ are shown in Nyquist plot form in Figure 3. Clearly, the errors involved in this example are non-trivial, and again from a control design point of view, the estimation near the cross-over frequency is far superior in Figure 3(b) under a direct estimation approach than it is in Figure 3(a) where indirect estimation is illustrated, and this difference in accuracy is clearly predicted by the theoretical and empirical results of Figure 2 which have already been discussed.

Turning now to the examination of joint input-output methods, consider the 'basic' joint inputoutput method described in $\S 4.3 .1$. Then again employing Monte-Carlo simulation to estimate the true variability of the ensuing estimate by averaging over 1000 data and subsequent estimate realisations, the results of this are shown as the solid line in Figure 4. Also shown on that Figure as a dashed line is the new quantification (56) which, via its very close match to the solid line showing the 'true' variability, is illustrated to be an accurate approximation. In order to aid comparison, the previously shown quantifications (24), (37) for direct and basic indirect method, whose accuracy was established in the previous Figure 2, are also provided in Figure 4.

A main point to notice is the qualitative and quantitative difference between these direct, and basic indirect variances when compared to the basic joint input-output method variability shown as the solid line, which further substantiates the theme of this paper that it is not generically true that all indirect estimation methods offer equivalent accuracy when compared to direct estimation methods.

In order to illustrate the results obtained here for the co-prime factor method, consider the use of 
the pre-filter

$$
X(q)=\frac{1}{q^{2}-1.8237 q+0.9801}
$$

which is of bandpass type with centre frequency at $0.4 \mathrm{rad} / \mathrm{s}$. In this case, the true frequency response variability $\operatorname{Var}\left\{G_{\mathrm{co}}\left(e^{j \omega}, \widehat{\theta}_{N}, \widehat{\beta}_{N}\right)\right\}$, again as estimated via Monte-Carlo simulation over 1000 data realisations, is shown as the solid line in Figure 5. Almost exactly matching this is the dashed line which is the new quantification (70). Note the presence of the peak at $0.4 \mathrm{rad} / \mathrm{s}$ introduced, as predicted, by the associated poles of $X(z)$. Note also, the significant difference (an order of magnitude at some frequencies) between the accuracy of the co-prime factor approach and that of the direct, and basic indirect approaches also shown as the indicated dashed and dash-dot lines in Figure 5.

Finally, the dual Youla method is examined by choosing the nominal model $G^{0}(q)$ as

$$
G^{0}(q)=\frac{0.0395 q-0.0099}{q^{2}-1.8 q+0.81}
$$

and the co-prime factors of $G^{0}(q)$ and $K(q)$ are taken according to the simple choice of $E_{G}(z)=$ $E_{K}(z)=1$. Using the controller (71) then implies that

$$
A_{c}^{0}(z)=\left(1-\rho_{1} z^{-1}\right)\left(1-\rho_{2} z^{-1}\right), \quad \rho_{1}=0.99 e^{j 0.2}, \rho_{2}=\overline{\rho_{1}}
$$

The open and closed loop poles $\left\{\xi_{k}\right\}$ and $\left\{\eta_{k}\right\}$ are as discussed previously, and combined with (72) and (50) lead to a quantification shown as the upper dashed line in Figure 6. Note that it agrees almost exactly with the solid line which is, as before, the true variability as estimated in Monte-Carlo fashion over 1000 data realisations.

Also shown in Figure 6 for comparison, are the quantifications (37) and (24) for direct and indirect methods, which have previously been shown to be essentially exact, and which are clearly different both quantitatively and qualitatively to the variance obtained via the dual Youla method.

Note that in this final simulation, the variance of $\left\{e_{t}\right\}$ has been reduced from the value used in previous simulations of $\sigma^{2}=10$, to $\sigma^{2}=0.1$, and in order to illustrate how the difference between direct and indirect methods depends upon the size of this variance relative to the variance $\mu$ of the reference $\left\{r_{t}\right\}$.

In particular, note that the difference between direct and basic indirect methods shown in Figure 6 is such that when $\sigma^{2}=0.1$, indirect methods can provide smaller relative variances at some frequencies, and as shown in Figure 2, this is not the case when $\sigma^{2}=10$.

This indicates that it is not possible to make any claims about the generic superiority of direct, versus indirect methods from a point of view that concentrates on accuracy at specific frequencies. On the other hand, an important issue not mentioned so far is that by virtue of its formulation, the weighting term $\kappa(\omega)$ defined in (20) has the property that

$$
\frac{1}{2 \pi} \int_{-\pi}^{\pi} \kappa(\omega) \mathrm{d} \omega=m
$$

where $m$ are the number of zeros $\left\{\xi_{1}, \cdots, \xi_{m}\right\}$ that define $\kappa(\omega)$, and (73) holds regardless of the location of these zeros.

This implies a 'waterbed' effect on frequency domain accuracy. Since the frequency domain average of $\kappa(\omega)$ (which is a major contributor to the total variance) is equal to a constant determined by the model orders employed, then any decrease in variance at one frequency, must be offset by an increase at another frequency. This is clearly illustrated in Figures 2-6, where any relative superiority of indirect versus direct methods at one frequency, is traded off by relative inferiority (the resonant peaks) at other frequencies. 


\section{Conclusion}

This paper has drawn on new quantifications for the variance of system estimates in the frequency domain that do not depend on an assumption of model order tending to infinity. This has allowed a study of the relationship between the accuracy of a variety of direct and indirect estimation methods that applies for the case of finite, and possibly low model order.

A key conclusion to arise from this work is that it is not generally true that there are negligible differences between the accuracy of the various methods. Furthermore, the accuracy of various indirect methods is not invariant to closed loop configuration, or to user choices such as that of pre-filter (co-prime factor method) or nominal system (dual Youla method).

In seeking to address these issues in a manner that is exact with respect to the finite model order chosen, certain assumptions of white set point signal, white measurement noise, and a certain controller structure have been imposed. A useful topic for further study would be to examine how these constraints might be lifted.

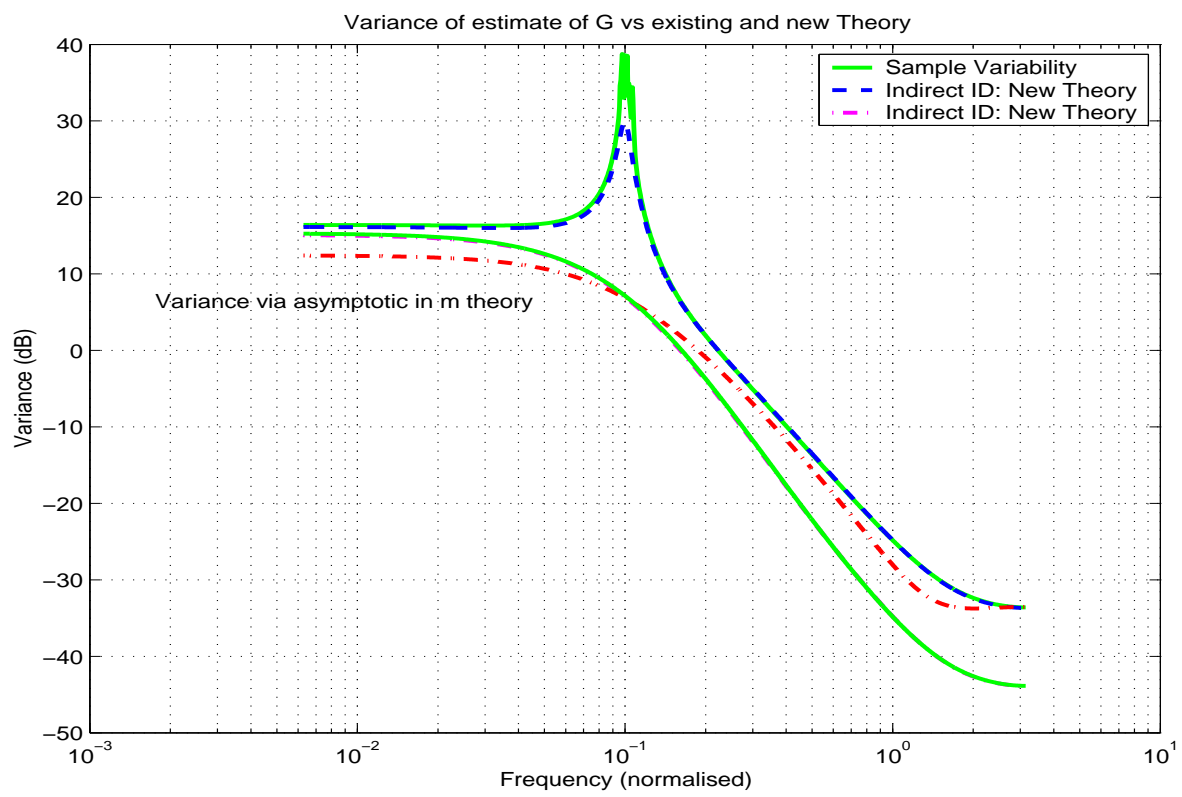

Figure 2: Variability of estimate obtained via direct and indirect identification. Solid lines are true variabilities, upper dashed and dash dot lines are new theoretical quantifications of those same variabilities. Lower dash dot line is pre-existing quantification. Note that the lower solid line, which is the sample variability for a direct method, is matched so closely by the new quantification (24) that is obliterates it. 


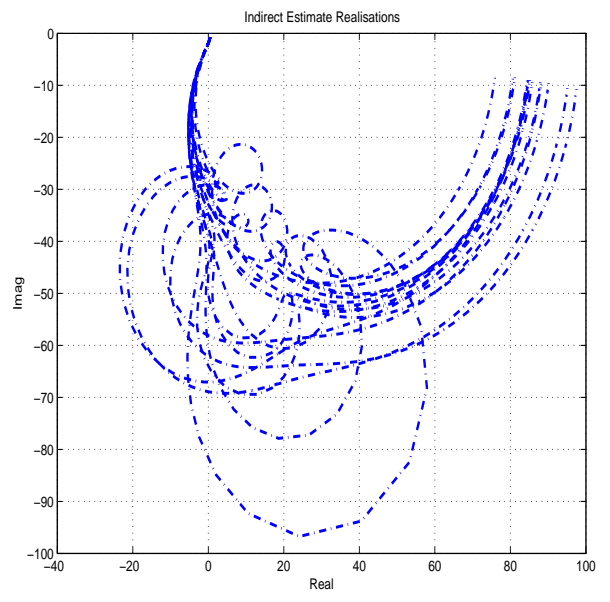

(a) Indirect Estimation

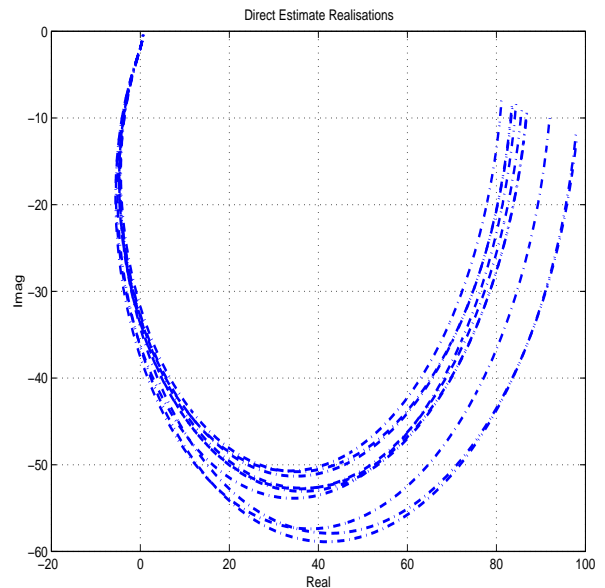

(b) Direct Estimation

Figure 3: First twenty realisations of estimates obtained via direct and indirect identification.

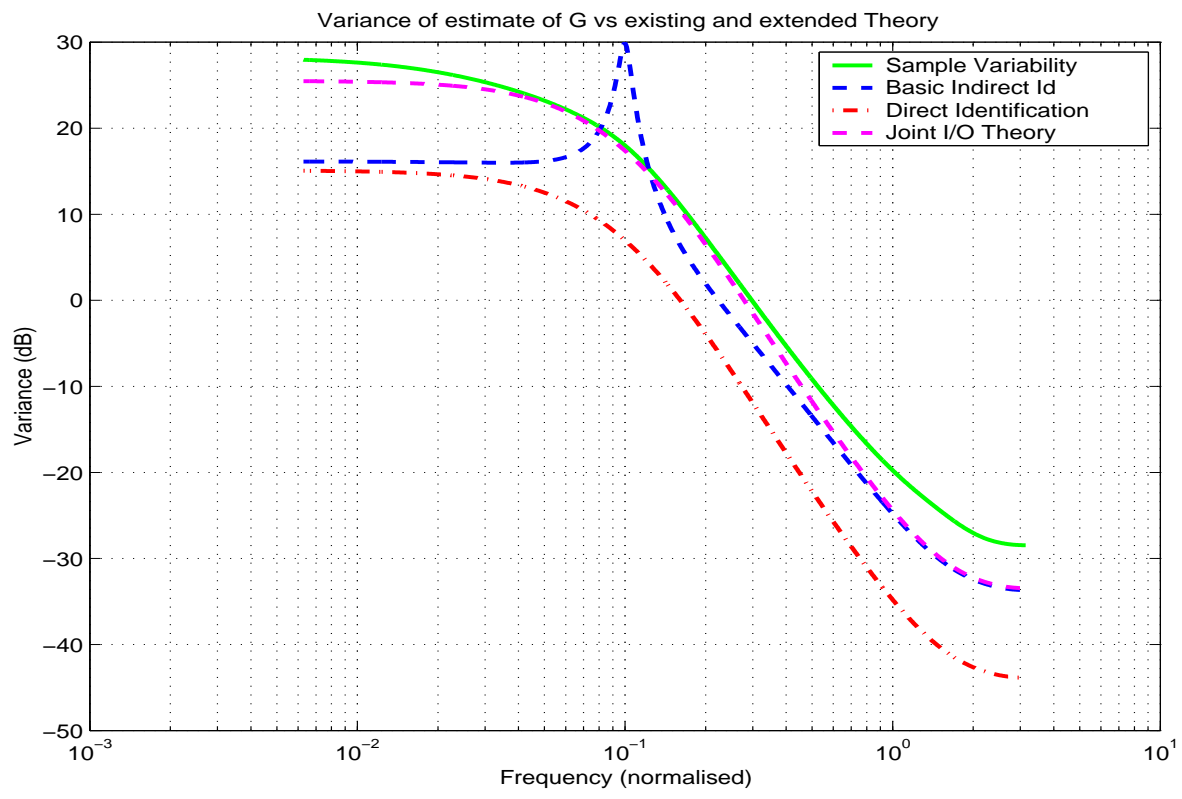

Figure 4: Variability of joint input-output method compared with direct and indirect approaches under the same experimental conditions. 


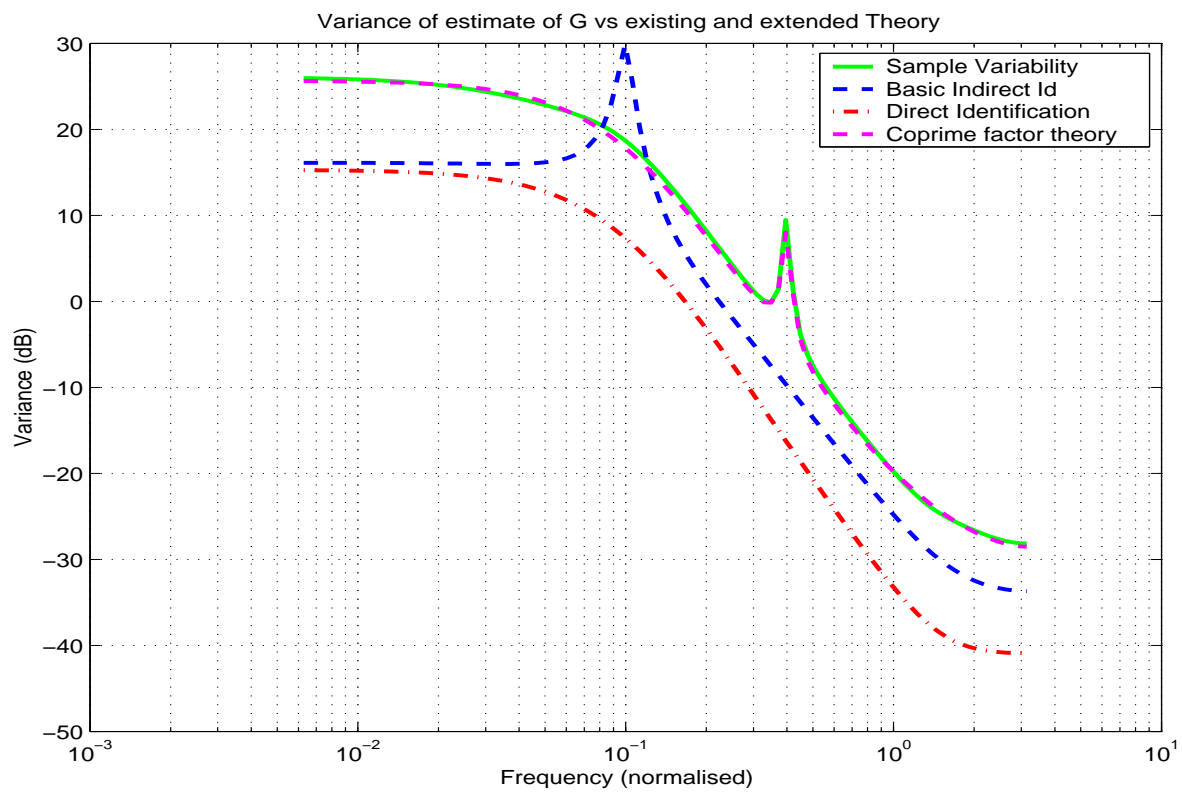

Figure 5: Variability of co-prime factor method estimate compared with direct and indirect approaches under the same experimental conditions

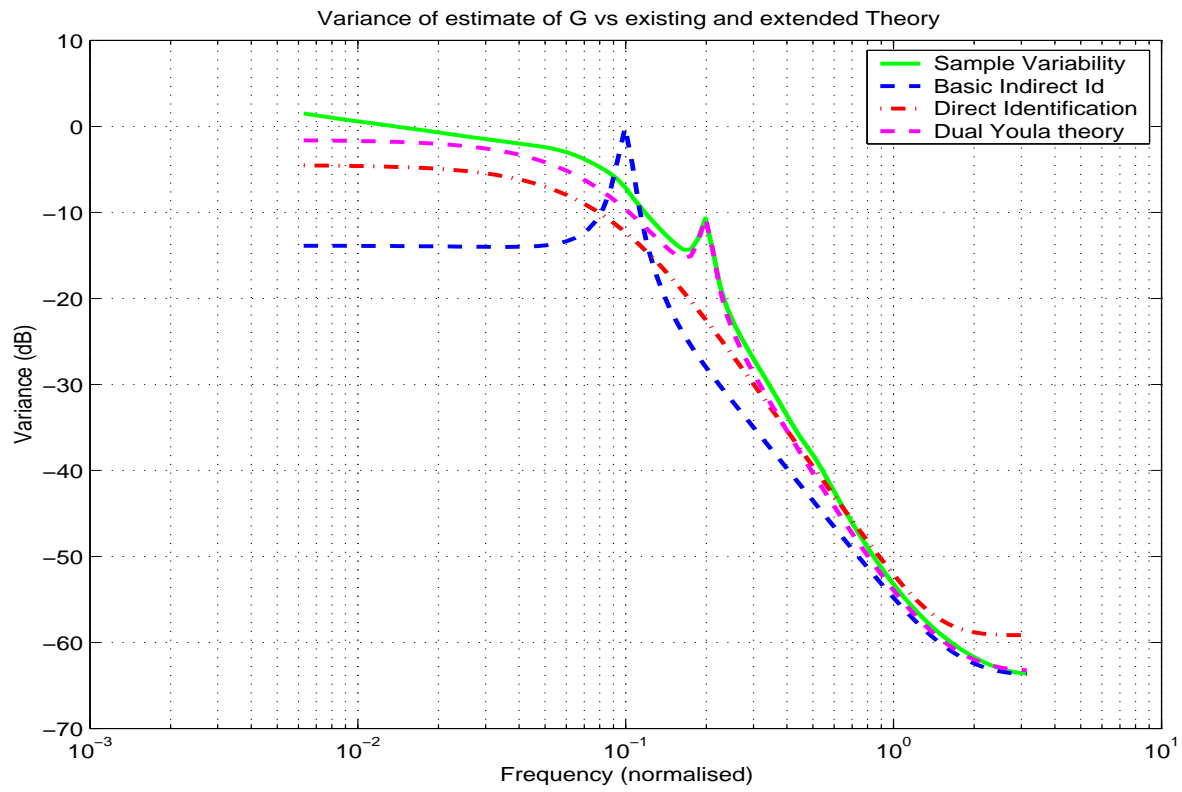

Figure 6: Variability of dual Youla method compared with direct and indirect approaches under the same experimental conditions 


\section{A Proof of Theorems}

\section{A.1 Proof of Theorem 4.1}

Proof. Note that according to (4), (8) (9)

$$
\Phi_{u}(\omega)=\left|\frac{A\left(e^{j \omega}\right)}{A_{c}\left(e^{j \omega}\right)}\right|^{2}\left[\mu+\sigma^{2}\right] .
$$

Therefore $F(z)=A(z) / A_{c}(z)$ and $H(z)=1$ substituted into (19) implies an associated $A_{\dagger}(z)$ given as

$$
A_{\dagger}(z)=A^{2}(z) \frac{A_{c}(z)}{A(z)}=A(z) A_{c}(z)
$$

which, as required by Theorem 3.1 is a polynomial. Application of that Theorem then completes the proof.

\section{A.2 Proof of Theorem 4.2}

Proof. Since $\Phi_{r}(\omega)=\mu$, it has spectral factor $F(z)=1$, and then according to the controller design assumption (10), the limiting estimate $S\left(q, \theta_{\circ}^{n}\right)$ can be expressed as

$$
S\left(q, \theta_{\circ}\right)=S(q)=\frac{A(q) L(q)}{P(q)(\widetilde{A}(q) L(q)+B(q))}=\frac{\widetilde{A}(q) L(q)}{A_{c}(q)} .
$$

Substituting this into (19) then implies

$$
A_{\dagger}(z)=A_{c}^{2}(z) \frac{L(z) \widetilde{A}(z)}{A_{c}(z)}=A_{c}(z) \widetilde{A}(z) L(z)
$$

which, as required by Theorem 3.1, is a polynomial. Therefore, according to that theorem

$$
\lim _{N \rightarrow \infty} N \operatorname{Var}\left\{T\left(e^{j \omega}, \widehat{\theta}_{N}\right)\right\}=\sigma^{2} \frac{\left|S\left(e^{j \omega}, \theta_{\circ}\right)\right|^{2}}{\mu} \kappa_{\mathrm{id}}(\omega)
$$

where $\kappa_{\mathrm{id}}(\omega)$ is given via (20) with the $\left\{\xi_{k}\right\}$ being the zeros of $z^{m_{\alpha}+m_{\beta}} A_{c}(z) \widetilde{A}(z) L(z)$. Application of (34), (35) then completes the proof.

\section{A.3 Proof of Theorem 4.3}

Proof. First note that according to the parameterisation (41)

$$
\begin{aligned}
\left.\frac{\mathrm{d} T(q, \theta)}{\mathrm{d} \theta}\right|_{\theta=\theta_{\circ}} & =\left[\frac{K(q)}{1+K(q) G\left(q, \theta_{\circ}\right)}-\frac{K^{2}(q) G\left(q, \theta_{\circ}\right)}{\left(1+K(q) G\left(q, \theta_{\circ}\right)\right)^{2}}\right] \frac{\mathrm{d} G\left(q, \theta_{\circ}\right)}{\mathrm{d} \theta} \\
& =K(q) S^{2}(q) \frac{\mathrm{d} G\left(q, \theta_{\circ}\right)}{\mathrm{d} \theta} .
\end{aligned}
$$

Therefore, from a variance point of view, estimation using the model structure (30), (41) is equivalent [10] to that associated with the model structure

$$
y_{t}=G(q, \theta) x_{t}+H(q, \theta) e_{t}, \quad x_{t} \triangleq K(q) S^{2}(q) r_{t} .
$$


In order to apply Theorem 3.1, and under the assumption of this theorem that $H(q)=1, \widetilde{A}(q) P(q)=$ $A(q)$ it is then necessary to consider

$$
A_{\dagger}(z)=\frac{A^{2}(q) S(q)}{K(q) S^{2}(q)}=\frac{A^{2}(q)}{K(q) S(q)}=\frac{A^{2}(q) A_{c}(q) L(q)}{\widetilde{A}(q) L(q) P(q)}=A_{c}(q) A(q)
$$

which, as required by Theorem 3.1, is a polynomial in $q^{-1}$. Application of Theorem 3.1 and recognising that

$$
\frac{\left|S\left(e^{j \omega}\right)\right|^{2}}{\Phi_{x}(\omega)}=\frac{1}{\left|S\left(e^{j \omega}\right) K\left(e^{j \omega}\right)\right|^{2} \mu}=\frac{1}{\Phi_{u}^{r}(\omega)}
$$

then completes the proof.

\section{A.4 Proof of Theorem 4.4}

Proof. First, note that under the assumption of using the controller (10) and of $H(q)=1$

$$
\begin{gathered}
x_{t}=F(q) r_{t}, \quad F(q) \triangleq K \frac{E_{G}(q) P(q) L(q)}{E_{K}(q) A_{c}^{0}(q)} \\
W\left(q, \theta_{\circ}\right)=\frac{\widetilde{A}(q) L(q)}{A_{c}(q)}, \quad A_{c}(q) \triangleq \widetilde{A}(q) L(q)+K B(q) \\
R\left(q, \theta_{\circ}\right)=Y^{-1}(q) M(q) S(q)\left[G(q)-G^{0}(q)\right]=\frac{E_{K}(q)}{E_{G}(q)} \frac{B\left(q, \theta_{\circ}\right) A^{0}(q)-A\left(q, \theta_{\circ}\right) B^{0}(q)}{P(q) A_{c}(q)} .
\end{gathered}
$$

Therefore, in order to use Theorem 3.1, it is necessary to consider

$$
\begin{aligned}
A_{\dagger}(q) & =E_{G}^{2}(q) P^{2}(q) A_{c}^{2}(q) \frac{W\left(q, \theta_{\circ}\right)}{F(q)} \\
& =E_{G}^{2}(q) P^{2}(q) A_{c}^{2}(q) \frac{\widetilde{A}(q) L(q)}{A_{c}(q)} \frac{A_{c}^{0}(q) E_{K}(q)}{E_{G}(q) P(q) L(q)} \\
& =E_{G}(q) E_{K}(q) A(q) A_{c}(q) A_{c}^{0}(q) .
\end{aligned}
$$

As required by Theorem 3.1, this resultant $A_{\dagger}(z)$ is a polynomial in $z^{-1}$, and by assumption it is of order less that $m_{\gamma}+m_{\lambda}$. Therefore, Theorem 3.1 may be used to assert that with $\kappa_{\text {dy }}(\omega)$ defined in (20) with associated zeros $\left\{\xi_{k}\right\}$ being those of (49)

$$
\begin{aligned}
\lim _{N \rightarrow \infty} N \operatorname{Var}\left\{R\left(e^{j \omega}, \widehat{\theta}_{N}\right)\right\} & =\sigma^{2} \frac{\left|W\left(e^{j \omega}, \theta_{\circ}\right)\right|^{2}}{\Phi_{x}(\omega)} \kappa_{\mathrm{dy}}(\omega) \\
& =\frac{\sigma^{2}}{\Phi_{r}(\omega)}\left|S\left(e^{j \omega}\right)\right|^{2}\left|\frac{A^{0}\left(e^{j \omega}\right) E_{K}\left(e^{j \omega}\right)}{E_{G}\left(e^{j \omega}\right) P\left(e^{j \omega}\right) S^{0}\left(e^{j \omega}\right)}\right|^{2} \kappa_{\mathrm{dy}}(\omega)
\end{aligned}
$$

Furthermore,

$$
\mathrm{d} G\left(e^{j \omega}\right)=\frac{E_{G}\left(e^{j \omega}\right)}{E_{K}\left(e^{j \omega}\right)} \frac{L\left(e^{j \omega}\right) S^{0}\left(e^{j \omega}\right)}{A^{0}\left(e^{j \omega}\right) S^{2}\left(e^{j \omega}\right)} \mathrm{d} R\left(e^{j \omega}\right)
$$


and hence

$$
\begin{aligned}
\lim _{N \rightarrow \infty} N \operatorname{Var}\left\{G_{\mathrm{dy}}\left(e^{j \omega}, \widehat{\theta}_{N}\right)\right\} & =\frac{\sigma^{2}}{\Phi_{r}(\omega)}\left|S\left(e^{j \omega}\right)\right|^{2}\left|\frac{A^{0}\left(e^{j \omega}\right) E_{K}\left(e^{j \omega}\right)}{E_{G}\left(e^{j \omega}\right) P\left(e^{j \omega}\right) S^{0}\left(e^{j \omega}\right)}\right|^{2} \\
& \left|\frac{E_{G}\left(e^{j \omega}\right) L\left(e^{j \omega}\right) S^{0}\left(e^{j \omega}\right)}{E_{K}\left(e^{j \omega}\right) A^{0}\left(e^{j \omega}\right) S^{2}\left(e^{j \omega}\right)}\right|^{2} \kappa_{\mathrm{dy}}(\omega) \\
& =\frac{\sigma^{2}}{\Phi_{r}(\omega)}\left|\frac{1}{S\left(e^{j \omega}\right) K\left(e^{j \omega}\right)}\right|^{2} \kappa_{\mathrm{dy}}(\omega)=\frac{\sigma^{2}}{\Phi_{u}^{r}(\omega)} \kappa_{\mathrm{dy}}(\omega) .
\end{aligned}
$$

\section{A.5 Proof of Theorem $\mathbf{4 . 5}$}

Proof. Since $G=T / S K$ then

$$
\mathrm{d} G=\frac{1}{S K} \mathrm{~d} T-\frac{T}{(S K)^{2}} \mathrm{~d} S K=\frac{1}{S K}[\mathrm{~d} T-G \mathrm{~d} S K]
$$

and therefore

$$
\begin{aligned}
\operatorname{Var}\left\{G\left(e^{j \omega}, \widehat{\theta}_{N}\right)\right\}= & \frac{1}{|S K|^{2}}\left[\operatorname{Var}\left\{T\left(e^{j \omega}, \widehat{\theta}_{N}\right)\right\}+|G|^{2} \operatorname{Var}\left\{S K\left(e^{j \omega}, \widehat{\beta}_{N}\right)\right\}\right. \\
& \left.-2 \operatorname{Re}\left\{G\left(e^{j \omega}\right) \operatorname{Cov}\left\{S K\left(e^{j \omega}, \widehat{\beta}_{N}\right) T\left(e^{j \omega}, \widehat{\theta}_{N}\right)\right\}\right]\right\} .
\end{aligned}
$$

However, as already established in (A.1)

$$
\lim _{N \rightarrow \infty} N \operatorname{Var}\left\{T\left(e^{j \omega}, \widehat{\theta}_{N}\right)\right\}=\sigma^{2} \frac{\left|S\left(e^{j \omega}, \theta_{\circ}\right)\right|^{2}}{\mu} \kappa_{\mathrm{id}}(\omega) .
$$

where $\kappa_{\text {id }}(\omega)$ is defined by (20) with the $\left\{\xi_{k}\right\}$ being the zeros of $z^{m_{\alpha}+m_{\beta}} A_{c}(z) \widetilde{A}(z) L(z)$. Turning now to the quantification of $\operatorname{Var}\left\{T\left(e^{j \omega}, \widehat{\beta}_{N}\right)\right\}$, note that the assumption of $H(q)=1$ implies that the noise model in (53) is $S K$ and hence the corresponding $A_{\dagger}(z)$ in (19) is

$$
A_{\dagger}(z)=A_{c}^{2}(z) \frac{A(z)}{A_{c}(z)}=A_{c}(z) A(z)
$$

which, under the assumption of the theorem is a polynomial of order less than $m_{\delta}+m_{\rho}$. Therefore, Theorem 3.1 can be applied to quantify the variance of the estimate $S K\left(q, \widehat{\beta}_{N}\right)$ as

$$
\lim _{N \rightarrow \infty} N \operatorname{Var}\left\{S K\left(e^{j \omega}, \widehat{\beta}_{N}\right)\right\}=\sigma^{2} \frac{\left|S K\left(e^{j \omega}, \beta_{\circ}\right)\right|^{2}}{\mu} \kappa_{\mathrm{di}}(\omega)
$$

where the zeros defining $\kappa_{\mathrm{di}}(\omega)$ are those of $z^{m_{\rho}+m_{\delta}} A_{c}(z) A(z)$, and hence are the same as those used to in the case of direct identification studied earlier. Finally, via Lemma B.1

$$
\lim _{N \rightarrow \infty} N \operatorname{Cov}\left\{S K\left(e^{j \omega}, \widehat{\beta}_{N}\right) T\left(e^{j \omega}, \widehat{\theta}_{N}\right)\right\}=-\frac{\sigma^{2}}{\mu} K\left(e^{j \omega}\right)\left|S\left(e^{j \omega}\right)\right|^{2} \Delta(\omega) .
$$


Substituting these variance expressions into (A.3) then implies that

$$
\begin{aligned}
\lim _{N \rightarrow \infty} N \operatorname{Var}\left\{G\left(e^{j \omega}, \widehat{\theta}_{N}\right)\right\}= & \frac{\sigma^{2}}{\mu|S K|^{2}}\left[|S|^{2} \kappa_{\mathrm{id}}(\omega)+|T|^{2} \kappa_{\mathrm{di}}(\omega)+2|S|^{2} \operatorname{Re}\{G K \Delta\}\right] \\
= & |S|^{2} \frac{\sigma^{2}}{\Phi_{u}^{r}(\omega)} \kappa_{\mathrm{id}}(\omega)+|T|^{2}\left(\frac{\mu+\sigma^{2}}{\mu}\right) \frac{\sigma^{2}}{\Phi_{u}(\omega)} \kappa_{\mathrm{di}}(\omega)+ \\
& \frac{2 \sigma^{2}}{\Phi_{u}^{r}(\omega)} \operatorname{Re}\{T \bar{S} \Delta(\omega)\} .
\end{aligned}
$$

Noting that from Theorem 4.2

$$
\lim _{N \rightarrow \infty} N \operatorname{Var}\left\{G_{\text {id }}\left(e^{j \omega}, \widehat{\theta}_{N}\right)\right\}=\frac{\sigma^{2}}{\Phi_{u}^{r}(\omega)} \kappa_{\mathrm{id}}(\omega), \quad \lim _{N \rightarrow \infty} N \operatorname{Var}\left\{G_{\mathrm{di}}\left(e^{j \omega}, \widehat{\theta}_{N}\right)\right\}=\frac{\sigma^{2}}{\Phi_{u}(\omega)} \kappa_{\mathrm{di}}(\omega)
$$

then completes the proof.

\section{A.6 Proof of Theorem 4.6}

Proof. Following along the same lines as the proof of Theorem 4.5, since $G=N M^{-1}$ then

$$
\mathrm{d} G=\frac{1}{M} \mathrm{~d} N-\frac{N}{M^{2}} \mathrm{~d} M=\frac{1}{M}[\mathrm{~d} N-G \mathrm{~d} M]
$$

and therefore

$$
\begin{aligned}
\operatorname{Var}\left\{G\left(e^{j \omega}, \widehat{\theta}_{N}\right)\right\}= & \frac{1}{|M|^{2}}\left[\operatorname{Var}\left\{N\left(e^{j \omega}, \widehat{\theta}_{N}\right)\right\}+|G|^{2} \operatorname{Var}\left\{M\left(e^{j \omega}, \widehat{\beta}_{N}\right)\right\}\right. \\
& \left.-2 \operatorname{Re}\left\{G\left(e^{j \omega}\right) \operatorname{Cov}\left\{M\left(e^{j \omega}, \widehat{\beta}_{N}\right) N\left(e^{j \omega}, \widehat{\theta}_{N}\right)\right\}\right]\right\} .
\end{aligned}
$$

Considering first $\operatorname{Var}\left\{N\left(e^{j \omega}, \widehat{\theta}_{N}\right)\right\}$, note that

$$
N\left(q, \theta_{\circ}^{n}\right)=\frac{B(q) X_{D}(q)}{A_{c}(q) X_{N}(q)}, \quad S\left(q, \theta_{\circ}^{n}\right)=\frac{\widetilde{A}(q) L(q)}{A_{c}(q)}
$$

and therefore, for the purposes of employing Theorem 3.1, it is necessary to consider

$$
A_{\dagger}(z)=A_{c}^{2}(z) X_{N}^{2}(z) \frac{\widetilde{A}(z) L(z)}{A_{c}(z)} \frac{X_{D}(z)}{X_{N}(z)}=A_{c}(z) \widetilde{A}(z) L(z) X_{N}(z) X_{D}(z) .
$$

Clearly, this is a polynomial, which under the assumptions of the theorem is of order less than $m_{\alpha}+$ $m_{\beta}$. Hence, according to Theorem 4.1

$$
\lim _{N \rightarrow \infty} N \operatorname{Var}\left\{N\left(e^{j \omega}, \widehat{\theta}_{N}\right)\right\}=\sigma^{2} \frac{\left|S\left(e^{j \omega}, \theta_{\circ}\right)\right|^{2}}{\Phi_{x}(\omega)} \kappa_{\mathrm{id} x}(\omega)
$$

where $\kappa_{\mathrm{id} x}(\omega)$ is defined by (20) with the $\left\{\xi_{k}\right\}$ being the zeros of $z^{m_{\alpha}+m_{\beta}} A_{c}(z) \widetilde{A}(z) L(z) X_{N}(z) X_{D}(z)$. Turning now to the quantification of $\operatorname{Var}\left\{M\left(e^{j \omega}, \widehat{\beta}_{N}\right)\right\}$, note that

$$
M\left(q, \theta_{\circ}^{n}\right)=\frac{A(q) X_{D}(q)}{A_{c}(q) X_{N}(q)}, \quad S K\left(q, \theta_{\circ}^{n}\right)=\frac{A(q)}{A_{c}(q)}
$$


and therefore, for the purposes of employing Theorem 3.1, it is necessary to consider

$$
A_{\dagger}(z)=A_{c}^{2}(z) X_{N}^{2}(z) \frac{A(z)}{A_{c}(z)} \frac{X_{D}(z)}{X_{N}(z)}=A_{c}(z) A(z) X_{N}(z) X_{D}(z) .
$$

Again, this is a polynomial, which under the assumptions on of the theorem is of order less than $m_{\rho}+m_{\delta}$. Hence, according to Theorem 4.1

$$
\lim _{N \rightarrow \infty} N \operatorname{Var}\left\{M\left(e^{j \omega}, \widehat{\theta}_{N}\right)\right\}=\sigma^{2} \frac{\left|S K\left(e^{j \omega}, \theta_{\circ}\right)\right|^{2}}{\Phi_{x}(\omega)} \kappa_{\operatorname{dix} x}(\omega) .
$$

where $\kappa_{\text {di } x}(\omega)$ is defined according to (20) with the $\left\{\xi_{k}\right\}$ being the zeros of $z^{m_{\rho}+m_{\delta}} A_{c}(z) A(z) X_{N}(z) X_{D}(z)$. Compared to the proof of Theorem 4.5, the inclusion of the pre-filter $X(q)$ has, relative to the joint input-output approach, added the poles and zeros of $X(q)$ into the associated polynomials $A_{\dagger}(z)$ that quantify variance. With this in mind, it is straightforward to see that the proof Lemma B.1 can be adapted in the same fashion to conclude that

$$
\lim _{N \rightarrow \infty} N \operatorname{Cov}\left\{M\left(e^{j \omega}, \widehat{\beta}_{N}\right) N\left(e^{j \omega}, \widehat{\theta}_{N}\right)\right\}=-\frac{\sigma^{2}}{\Phi_{x}(\omega)} K\left(e^{j \omega}\right)\left|S\left(e^{j \omega}\right)\right|^{2} \Delta(\omega)
$$

where $\Delta(\omega)$ is again given by (58) but now with $\Delta_{1}(\lambda, \omega)$ and $\Delta_{2}(\lambda, \omega)$ being (respectively) the reproducing kernels for space spanned by the elements of $S^{-1}(z) X(z) \mathrm{d} N(z, \theta) / \mathrm{d} \theta$ and $S K^{-1}(z) X(z) \mathrm{d} M(z, \theta) / \mathrm{d} \theta$ and with respect to the measure $\mu \mathrm{d} \lambda$, and it is then straightforward to see that these are the same spaces $V_{\zeta}$ and $V_{\tau}$ considered in (60), (61) for the joint input-output method after they are augmented to include the poles and zeros of $X(z)$. Substituting (A.7), (A.6) and (A.5) into (A.4) then implies that

$$
\begin{aligned}
\lim _{N \rightarrow \infty} N \operatorname{Var}\left\{G\left(e^{j \omega}, \widehat{\theta}_{N}\right)\right\}= & \frac{\sigma^{2}}{|M|^{2} \Phi_{x}(\omega)}\left[|S|^{2} \kappa_{\mathrm{id} x}(\omega)+|T|^{2} \kappa_{\mathrm{di} x}(\omega)+2|S|^{2} \operatorname{Re}\{G K \Delta\}\right] \\
= & |S|^{2} \frac{\sigma^{2}}{\Phi_{u}^{r}(\omega)} \kappa_{\mathrm{id} x}(\omega)+|T|^{2}\left(\frac{\mu+\sigma^{2}}{\mu}\right) \frac{\sigma^{2}}{\Phi_{u}(\omega)} \kappa_{\mathrm{di} x}(\omega)+ \\
& \frac{2 \sigma^{2}}{\Phi_{u}^{r}(\omega)} \operatorname{Re}\{T \bar{S} \Delta(\omega)\} .
\end{aligned}
$$

Substitution of (28) and (68) into the above then completes the proof.

\section{B Technical Lemma}

Lemma B.1. Under the conditions of Theorem 4.5

$$
\lim _{N \rightarrow \infty} N \operatorname{Cov}\left\{S K\left(e^{j \omega}, \widehat{\beta}_{N}\right) T\left(e^{j \omega}, \widehat{\theta}_{N}\right)\right\}=-\frac{\sigma^{2}}{\mu} K\left(e^{j \omega}\right)\left|S\left(e^{j \omega}\right)\right|^{2} \Delta(\omega),
$$

where $\Delta(\omega)$ is defined in (58).

Proof. Via a standard first order Taylor expansion argument [7]

$$
\widehat{\theta}_{N}-\theta_{\circ} \approx R^{-1}\left(\theta_{\circ}\right) \frac{\mathrm{d}}{\mathrm{d} \theta} V_{N}\left(\theta_{\circ}, \beta_{\circ}\right)
$$


where the accuracy in the above approximation increases with increasing $N$ and

$$
R(\theta) \triangleq \lim _{N \rightarrow \infty} \frac{1}{N} \sum_{t=1}^{N} \mathbf{E}\left\{\psi_{t}(\theta) \psi_{t}^{T}(\theta)\right\}, \quad \frac{\mathrm{d}}{\mathrm{d} \theta} V_{N}\left(\theta_{\circ}, \beta_{\circ}\right)=-\frac{1}{N} \sum_{t=1}^{N} \psi_{t}\left(\theta_{\circ}\right) e_{t}
$$

with

$$
\psi_{t}\left(\theta_{\circ}\right) \triangleq S^{-1}\left(q, \theta_{\circ}\right)\left[\frac{\mathrm{d} T\left(q, \theta_{\circ}\right)}{\mathrm{d} \theta}, \frac{\mathrm{d} S\left(q, \theta_{\circ}\right)}{\mathrm{d} \theta}\right]\left[\begin{array}{c}
r_{t} \\
e_{t}
\end{array}\right]
$$

Similarly

$$
\widehat{\beta}_{N}-\beta_{\circ} \approx M^{-1}\left(\beta_{\circ}\right) \frac{\mathrm{d}}{\mathrm{d} \beta} V_{N}\left(\theta_{\circ}, \beta_{\circ}\right)
$$

where

$$
M(\beta) \triangleq \lim _{N \rightarrow \infty} \frac{1}{N} \sum_{t=1}^{N} \mathbf{E}\left\{\phi_{t}(\beta) \phi_{t}^{T}(\beta)\right\}, \quad \frac{\mathrm{d}}{\mathrm{d} \beta} V_{N}\left(\beta_{\circ}, \beta_{\circ}\right)=-\frac{1}{N} \sum_{t=1}^{N} \phi_{t}\left(\beta_{\circ}\right) e_{t}
$$

with

$$
\phi_{t}\left(\beta_{\circ}\right) \triangleq-S K^{-1}\left(q, \beta_{\circ}\right)\left[\frac{\mathrm{d} S K\left(q, \beta_{\circ}\right)}{\mathrm{d} \beta},-\frac{\mathrm{d} S K\left(q, \beta_{\circ}\right)}{\mathrm{d} \beta}\right]\left[\begin{array}{l}
r_{t} \\
e_{t}
\end{array}\right]
$$

Therefore,

$$
N \operatorname{Cov}\left\{\widehat{\theta}_{N} \widehat{\beta}_{N}^{T}\right\} \approx-\sigma^{2} R^{-1}\left(\theta_{\circ}\right) \frac{1}{N} \sum_{t=1}^{N} \mathbf{E}\left\{\psi_{t}\left(\theta_{\circ}\right) \phi_{t}^{T}\left(\beta_{\circ}\right)\right\} M^{-1}\left(\beta_{\circ}\right)
$$

with increasing accuracy in the approximation as $N \rightarrow \infty$. Now, defining

$$
\begin{array}{cc}
\Pi(q, \theta) \triangleq[T(q, \theta), S(q, \theta)], & \Gamma(q, \beta) \triangleq[S K(q, \beta), S K(q, \beta)] \\
Z\left(q, \theta_{\circ}\right) \triangleq\left[\frac{\mathrm{d} T\left(q, \theta_{\circ}\right)}{\mathrm{d} \theta}, \frac{\mathrm{d} S\left(q, \theta_{\circ}\right)}{\mathrm{d} \theta}\right], & W\left(q, \beta_{\circ}\right) \triangleq\left[\frac{\mathrm{d} S K\left(q, \beta_{\circ}\right)}{\mathrm{d} \beta},-\frac{\mathrm{d} S K\left(q, \beta_{\circ}\right)}{\mathrm{d} \beta}\right]
\end{array}
$$

then again using a first order Taylor expansion

$\Pi\left(e^{j \omega}, \widehat{\theta}_{N}\right)-\Pi\left(e^{j \omega}, \theta_{\circ}\right) \approx Z^{T}\left(e^{j \omega}, \theta_{\circ}\right)\left[\widehat{\theta}_{N}-\theta_{\circ}\right], \quad \Gamma\left(e^{j \omega}, \widehat{\beta}_{N}\right)-\Gamma\left(e^{j \omega}, \beta_{\circ}\right) \approx W^{T}\left(e^{j \omega}, \theta_{\circ}\right)\left[\widehat{\beta}_{N}-\beta_{\circ}\right]$.

Therefore, combining with (B.1) implies that

$$
\begin{gathered}
\lim _{N \rightarrow \infty} N \frac{1}{K\left(e^{j \omega}\right)\left|S\left(e^{j \omega}\right)\right|^{2}} \operatorname{Cov}\left\{\Gamma\left(e^{j \omega}, \widehat{\theta}_{N}\right) \Pi\left(e^{j \omega}, \widehat{\theta}_{N}\right)\right\}= \\
-\left[\frac{Z\left(e^{j \omega}, \theta_{\circ}\right)}{S\left(e^{j \omega}\right)}\right]^{\star} T_{n}^{-1}\left(\frac{Z \phi_{\zeta} Z^{\star}}{|S|^{2}}\right) T_{n}\left(\frac{Z \phi_{\zeta} W^{\star}}{|S|^{2} K^{\star}}\right) T_{n}^{-1}\left(\frac{W \phi_{\zeta} W^{\star}}{|S K|^{2}}\right) \frac{W\left(e^{j \omega}, \beta_{\circ}\right)}{S\left(e^{j \omega}\right) K\left(e^{j \omega}\right)}= \\
-\frac{1}{2 \pi} \int_{-\pi}^{\pi} \varphi(\lambda, \omega) \gamma(\omega, \lambda) \mu \mathrm{d} \lambda
\end{gathered}
$$

where

$$
\begin{gathered}
\varphi(\lambda, \omega)=\left[\frac{Z\left(e^{j \omega}, \theta_{\circ}\right)}{S\left(e^{j \omega}\right)}\right]^{\star} T_{n}^{-1}\left(\frac{Z \phi_{\zeta} Z^{\star}}{|S|^{2}}\right) \frac{Z\left(e^{j \lambda}, \theta_{\circ}\right)}{S\left(e^{j \lambda}\right)} \\
\gamma(\omega, \lambda)=\left[\frac{W\left(e^{j \lambda}, \beta_{\circ}\right)}{S\left(e^{j \lambda}\right) K\left(e^{j \lambda}\right)}\right]^{\star} T_{n}^{-1}\left(\frac{W \phi_{\zeta} W^{\star}}{|S K|^{2}}\right) \frac{W\left(e^{j \omega}, \beta_{\circ}\right)}{S\left(e^{j \omega}\right) K\left(e^{j \omega}\right)}
\end{gathered}
$$


Now, according to (15), then by construction $\varphi(\lambda, \omega)$ and $\gamma(\lambda, \omega)$ are diagonal $2 \times 2$ matrix-valued functions. Denote the top left elements of them as $\Delta_{1}(\lambda, \omega)$ and $\Delta_{2}(\lambda, \omega)$. Then, since $\Phi_{r e}(\omega)=0$

$$
\lim _{N \rightarrow \infty} N \frac{1}{K\left(e^{j \omega}\right)\left|S\left(e^{j \omega}\right)\right|^{2}} \operatorname{Cov}\left\{S K\left(e^{j \omega}, \widehat{\beta}_{N}\right) T\left(e^{j \omega}, \widehat{\theta}_{N}\right)\right\}=-\frac{1}{2 \pi} \int_{-\pi}^{\pi} \Delta_{1}(\lambda, \omega) \Delta_{2}(\omega, \lambda) \mu \mathrm{d} \lambda
$$

However, using the ideas developed in [10], $\Delta_{1}(\lambda, \omega)$ and $\Delta_{2}(\lambda, \omega)$ are both reproducing kernels for the space spanned by the elements of (respectively) of $S^{-1}(z) \mathrm{d} T(z, \theta) / \mathrm{d} \theta$ and $S K^{-1}(z) \mathrm{d} S K(z, \theta) / \mathrm{d} \theta$ and with respect to the measure $\mu \mathrm{d} \lambda$. Furthermore, these spaces are equal to those of $V_{\zeta}$ and $V_{\tau}$ defined in (60), (61) and hence again via the results in [10], the kernels can then be expressed via the quantities in (59) after division by $\mu$.

\section{References}

[1] U. Forssell And L. Ljung, Closed-loop identification revisited, Automatica, 53 (1999), pp. 1215-1241.

[2] M. Gevers, X. Bombois, B. Codrons, G. Scorletti, And B. Anderson, Model validation for control and controller validation in a prediction error identification framework. part $i$ : Theory, Automatica, 39 (2003), pp. 403-415.

[3] _ Model validation for control and controller validation in a prediction error identification framework. part ii: Illustrations, Automatica, 39 (2003), pp. 417-427.

[4] M. Gevers, L. LJUnG, AND P. VAN DEN HoF, Asymptotic variance expressions for closed loop identification, Automatica, 37 (2001), pp. 781-786.

[5] H. Hualmarsson, M. Gevers, and F. De Bruyne, For some model based control design criteria, closed loop identification gives better performance, Automatica, 32 (1996), pp. 16591673.

[6] E. Lehmann, Theory of Point Estimation, John Wiley \& Sons, 1983.

[7] L. LJung, System Identification: Theory for the User, (2nd edition), Prentice-Hall, Inc., New Jersey, 1999.

[8] L. LjUng AND U. Forssell, Variance results for closed loop identifiction methods, in Proceedings of the 36th IEEE Conference on Decision and Control, 1997, pp. 2435-2440.

[9] L.LuUng, Asymptotic variance expressions for identified black-box transfer function models, IEEE Transactions on Automatic Control, AC-30 (1985), pp. 834-844.

[10] B. NinnESS AND H. HJALMARSSON, Variance error quantifications that are exact for finite model order, Submitted to IEEE Transactions on Automatic Control, (2003).

[11] P.M.J. VAN DEN Hof AND R. Schrama, Identification and control-Closed loop issues, Automatica, 31 (1995), pp. 1751-1770.

[12] R. Schrama, Approximate Identification and Control Design with Application to a Mechanical System, PhD thesis, Delft University of Technology, 1992. 
[13] P. VAN DEN HoF, Closed-loop issues in system identification, Annual Reviews in Control, 22 (1998), pp. 173-186.

[14] L.-L. XIE AND L. LJUnG, Asymptotic variance expressions for estimated frequency functions, IEEE Transactions on Automatic Control, 46 (2001), pp. 1887-1899. 